\title{
Characterizing Dynamic Transitions Associated with Snap-Through of Clamped Shallow Arches
}

\author{
Yenny Chandra $^{\mathrm{a}}$, Richard Wiebe ${ }^{\mathrm{b}}$, Ilinca Stanciulescu ${ }^{\mathrm{a}, *}$, \\ Lawrence N. Virgin ${ }^{b}$, Stephen M. Spottswood ${ }^{c}$, Thomas G. Eason ${ }^{\mathrm{c}}$ \\ ${ }^{a}$ Rice University, Department of Civil and Environmental Engineering, \\ Houston, TX, 77005, U.S.A \\ ${ }^{b}$ Duke University, School of Engineering, Durham, NC, 27708, U.S.A. \\ ${ }^{c}$ Air Force Research Laboratory, Structural Sciences Center, \\ 2790 D. Street, WPAFB, OH, U.S.A.
}

\begin{abstract}
Slender curved structures can often be found as components of complex structures in civil, mechanical, and aerospace systems. Under extreme loadings, a curved structure might undergo snap-through buckling, i.e., the structure is forced to its inverted configuration, inducing fatigue. Therefore, it is important to identify the stability boundaries of structures and to obtain an accurate description of their performance if the response moves beyond those boundaries. In this paper, a combined experimental - computational framework is used to analyze the transient behavior of clamped-clamped shallow arches. We examine, both experimentally and using Finite Element Analysis (FEA), the response of shallow arches under harmonic distributed loading. Various types of responses are identified and regions in the forcing parameter space that lead to snap-through and chaotic responses are determined.
\end{abstract}

Keywords: snap-through, curved structures, transient loads, stability boundaries

\section{Introduction}

The performance demands of next generation aircraft has lead to increasingly lighter designs that require slender structural components. Such

*Corresponding author. Tel.: +1713 348 4704; fax: +1713 3485268.

Email address: ilinca.s@rice.edu (Ilinca Stanciulescu) 
systems are susceptible to instabilities, particularly buckling. A recent report by the Air Force Research Lab (AFRL) [1] recognizes the importance of understanding the fundamental instability behavior of slender curved structures. This requires both the identification of the stability boundaries of such structures and the accurate description of the performance of the structures if the response moves beyond those boundaries.

In this paper, we present (1) a combined experimental - computational framework for the analysis of large (non-linear) structural deformations and for the assessment of stability in slender structural components and (2) provide a methodology to determine the boundaries of the stability regions in the parameter space under consideration. The instability of interest in this paper is snap-through buckling, in which a curved structure is forced to its inverted configuration (Figure 1 (a)). Snap-through buckling occurs as the consequence of two factors, first, the destabilization, or more often the disappearance of, an equilibrium position under the change of a system parameter, and second, the existence of another stable equilibrium configuration at a remote location in the state space (Figure 1 (b)). In this sense snap-through buckling is a global dynamic transition as the result of a local static instability [2].

The definition of what is, and what is not, a snap-through event in a dynamic response can be elusive [3]. However, the static equilibria act as an organizing framework for the dynamic response and are used in the following work as a guide to aid in identifying dynamic snap-through.

Multiple studies have been performed on the dynamic behavior of shallow

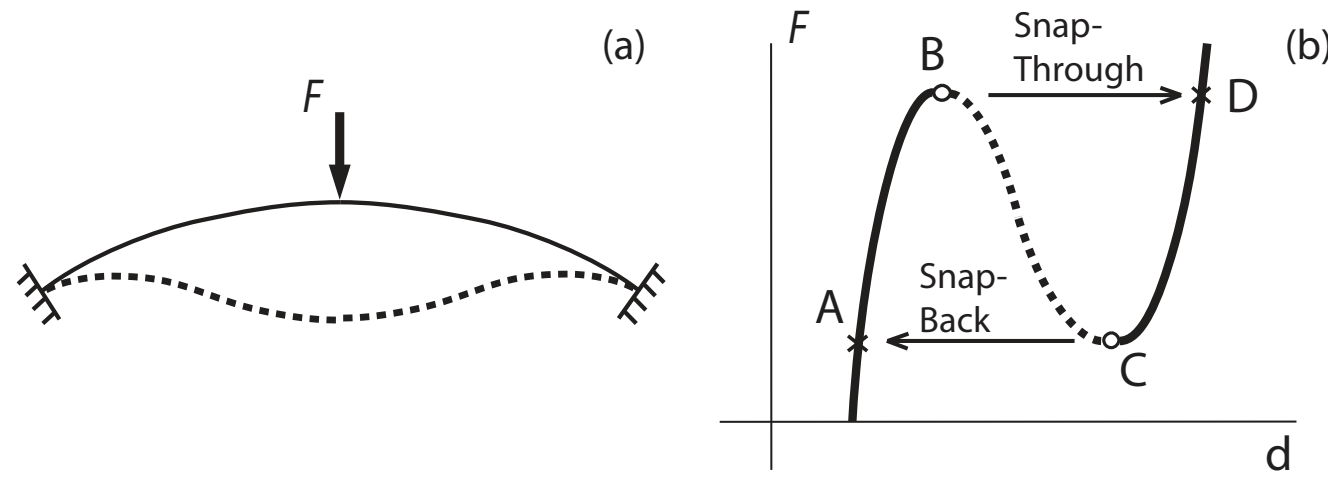

Figure 1: Snap-through buckling of shallow structures: (a) initial and post-snap configurations, (b) force-displacement curve. 
curved structures. The behavior of elastic shallow structures under highfrequency harmonic loading is presented by Huang [4]. Poon [5] perfomed a numerical study of snap-through on a clamped-clamped buckled initially flat beam under harmonic excitation. Ehrhardt [6] studied the response of a clamped-clamped arch subjected to swept sine testing. Parks [7] reported the effects of geometric imperfections and boundary conditions of curved arch structures. Murphy [8] et al. discussed experiments on thermally loaded clamped panels. $\mathrm{Ng}$ [9] presented experimental results of buckled curved panels subjected to intense acoustic excitation. A more thorough discussion of previous work on shallow arches and panels can be found in [2].

In previous work [10], static and dynamic snap-through of a single-degreeof-freedom (SDOF) arch was investigated, both experimentally and through numerical simulations. The SDOF arch provided an ideal first step as it exhibits both snap-through and chaos, but still allows for relatively unambiguous experimental and numerical verification without the complexities involved in distributed systems. The paper showed that it is possible to accurately model dynamic snap-through of real systems, however, the SDOF system is obviously limited in spatial complexities of real structures such as aircraft panels. The curved beam offers the next step up in difficulty and fidelity. In [11], we presented a numerical investigation of curved beams with a specific configuration and loading where a simplified model can be established. Several tools and measures useful to assess the structure's ability to survive post-snap-through event were presented. This framework is employed in the current work to characterize the transient response of experimental curved beams accounting for asymmetry and imperfections.

The curved arches used in the experimental study presented in Section 2 had fabrication imperfections and were also subjected to prestressing caused by the clamping procedure, two effects which cannot be properly captured in modal analysis techniques as those discussed by [12]. For this reason the numerical modeling was done using Finite Element Analysis (FEA). We use the Finite Element Analysis Program (FEAP), a research code that includes most commonly used finite elements and solvers and provides a reliable framework for developing and implementing new user formulations [13].

This paper is organized as follows. In Section 2, we present the experimental setup and describe the geometry and material properties of the structure under consideration. In Section 3, we discuss the static analysis of the shallow arches under both point and distributed loading. Owing to the difficulty of performing displacement control with a distributed loading, the 
experimental statics results are all for a midspan point load. The experimental results are then compared with, and used to verify, those obtained via FEA. Finally the FEA is extended to distributed loading. Section 4 analyzes the dynamic response of the structures under harmonic excitations and the snap-through boundaries in the parameter space are determined. A summary of our findings is presented in the concluding section.

\section{Experimental Setup}

The arches used in the experiment were fabricated based on a design of a circular arch with radius of $3048 \mathrm{~mm}$ and an arc length of $381.25 \mathrm{~mm}$, with the intention of having a horizontal projection of $304.8 \mathrm{~mm}$. A photograph of the experimental arch is shown in Figure 2. The arch was clamped to the white circular drum of the shaker which provided the distributed dynamic load via the inertia of the arch itself. For the static testing the shaker (turned off) and used only as a mount for the arch. The static loading mechanism consisted of the point loading coupler attached to an adjustable threaded rod through a load cell. The threaded rod was moved perpendicular to the arch to displace the structure, while the load cell provided the corresponding point load magnitude. Note that only compressive forces were accessible as the loading was provided through a pushing contact. The static arch displacements were measured by Digital Image Correlation (DIC) [14], which necessitated the speckle pattern visible in the photo. For the dynamic experiments, both DIC and a laser vibrometer [14] were used. The laser vibrometer was necessary as the DIC system was limited in runtime, and also provided a level of verification as it was independent of the DIC system.

The geometry of the experimental arch is given in Table 1 in $12.7 \mathrm{~mm}$ $\left(1 / 2^{\prime \prime}\right)$ intervals, which correspond to the location of the pieces of reflective tape in Figure 2. The measurements $y_{u c}$ and $y_{c}$ correspond to the pre- and post-clamped configurations respectively, as shown in Figure 3. The configuration changed when the arch was mounted to the shaker because the initial length and curvature of the arch at the ends do not exactly match that of the clamps, which had pre-drilled holes for screws and were tightened to a prescribed torque. These differences, although small, induce internal prestresses, and lead to a very noticeable difference between $y_{u c}$ and $y_{c}$. The cross-section and material properties of the arch are given in Table 2. 


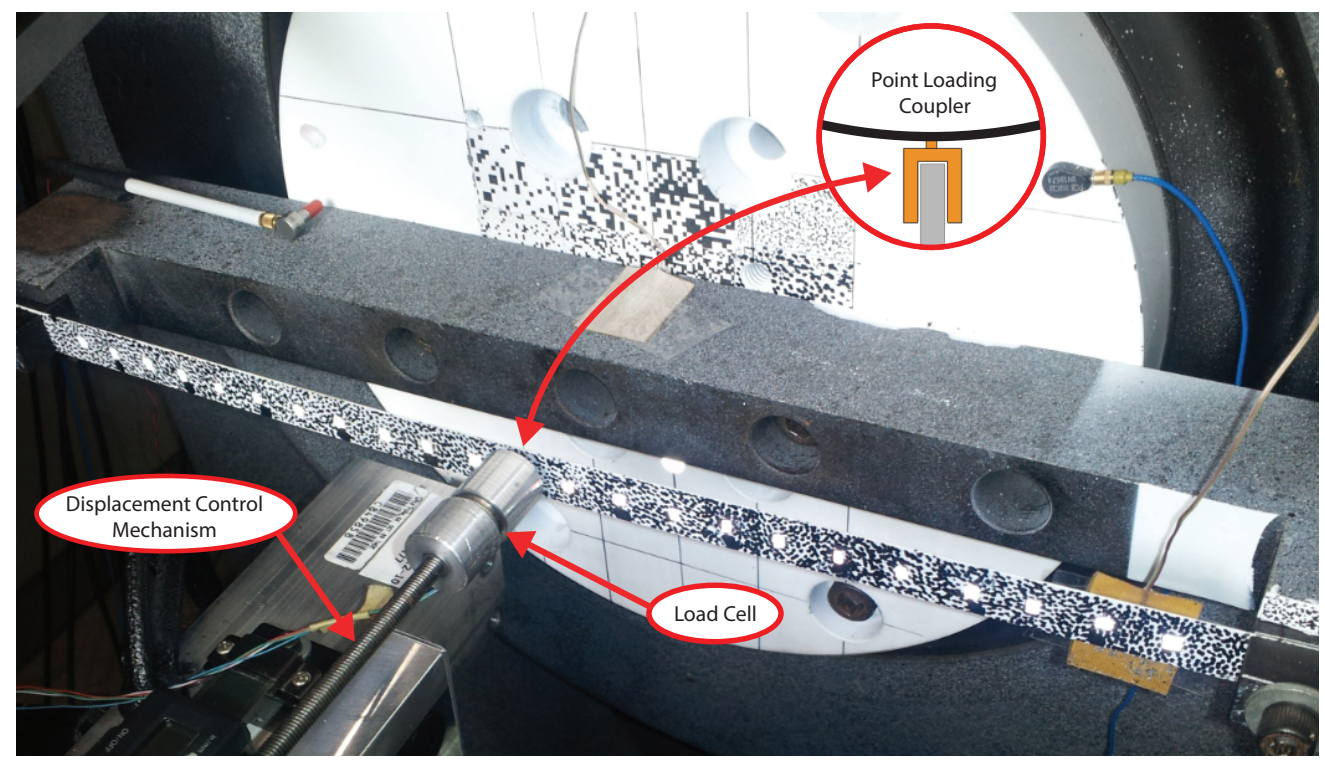

Figure 2: Photograph of curved arch attached to shaker in clamped-clamped configuration; point loading mechanism used for static testing also visible.
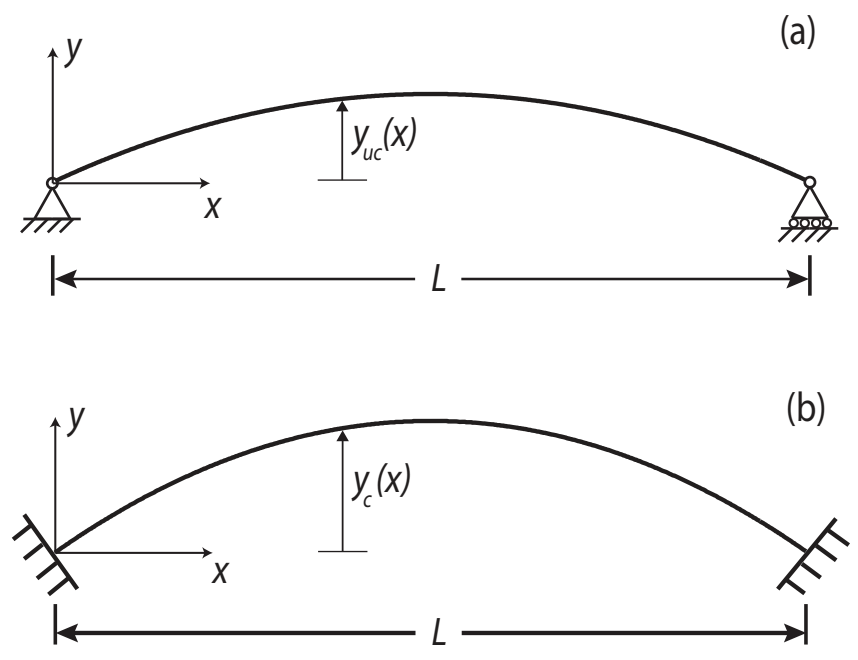

(c)

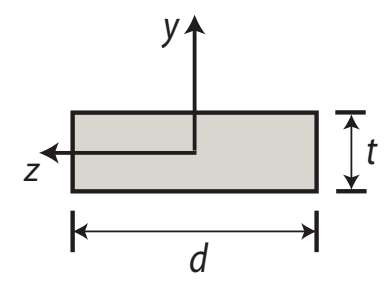

Figure 3: Schematic of curved arch geometry: (a) pre-clamping geometry, (b) postclamping geometry, and (c) cross-section. 
Table 1: Geometry (mm)

\begin{tabular}{||l|c|c|c|c|c|c|c|c|c|c|c|c||}
\hline$x$ & 0.0 & 12.7 & 25.4 & 38.1 & 50.8 & 63.5 & 76.2 & 88.9 & 101.6 & 114.3 & 127.0 & 139.7 \\
\hline$y_{u c}$ & 0.000 & 0.523 & 0.858 & 1.155 & 1.352 & 1.518 & 1.695 & 1.820 & 1.923 & 2.001 & 2.090 & 2.138 \\
\hline$y_{c}$ & 0.000 & 0.447 & 0.849 & 1.235 & 1.632 & 1.990 & 2.365 & 2.666 & 2.916 & 3.168 & 3.350 & 3.440 \\
\hline \hline 152.4 & 165.1 & 177.8 & 190.5 & 203.2 & 215.9 & 228.6 & 241.3 & 254.0 & 266.7 & 279.4 & 292.1 & 304.8 \\
\hline 2.147 & 2.159 & 2.085 & 1.959 & 1.838 & 1.713 & 1.655 & 1.521 & 1.348 & 1.118 & 0.858 & 0.543 & 0.000 \\
\hline 3.433 & 3.363 & 3.245 & 3.043 & 2.813 & 2.483 & 2.210 & 1.882 & 1.564 & 1.208 & 0.853 & 0.471 & 0.000 \\
\hline
\end{tabular}

Table 2: Cross Section and Material Properties

\begin{tabular}{|l|r|}
\hline Properties & Values \\
\hline Width d $[\mathrm{mm}]$ & 12.7 \\
Thickness t $[\mathrm{mm}]$ & 0.61 \\
Young's Modulus $\left[\mathrm{N} / \mathrm{mm}^{2}\right]$ & 206,843 \\
Poisson's Ratio & 0.28 \\
Density $\left[\mathrm{kg} / \mathrm{mm}^{3}\right]$ & $7.83 \cdot 10^{-6}$ \\
\hline
\end{tabular}

\section{Static Equilibria and Stability}

In this section, we first present the static force-displacement diagram, obtained both experimentally and using numerical approaches, for the arch under point loading. Then, the FEA is extended to obtain the equilibrium path under distributed loading. The numerical simulations are performed with a large deformation beam formulation based on the Euler-Bernoulli theory. Based on a mesh refinement study, it was decided to use 385 elements.

In order to properly match the experimental results, a prestress was applied to the FEA model. During the course of the experiments it was observed that when clamping the structure, a slight compressive end force was required to fit it in the clamps, and that when tightened, the clamps would rotate the ends downward. We therefore decided to match the prestress numerically by using an axial force and end moments in two prestressing stages as illustrated in Figure 4 (a). The experimentally measured initial unclamped configuration, $y_{u c}$, is used as the initial shape in the FEA analysis. In the first stage, the arch was assumed to have pin-roller boundary conditions (BC's) and it is subjected to an end compressive force $F_{p}$. Following this, the roller on the right side is replaced with a pin, and bending moments $M_{p}$ are applied at each end in the second stage. Finally, the pinned BC's are replaced by clamped BC's, taking care not to relieve the generated internal forces in 
the FEA model. The Euclidean error norm, $\left\|y_{F E A}-y_{c}\right\|$, between the FEA shape after prestressing, $y_{F E A}$, and the experimentally measured clamped configuration, $y_{c}$, is computed for different values of $F_{p}$ and $M_{p}$. Figure $4(\mathrm{~b})$ shows the contour plot of the error with a minimum at $F_{p}=1.628 \mathrm{~N}$ and $M_{p}=0.029 \mathrm{~N} \cdot \mathrm{m}$. The shape of the arch after stage 1 and 2 of this prestress case is shown in Figure 4 (c) (note that the "y" axis is heavily exaggerated).

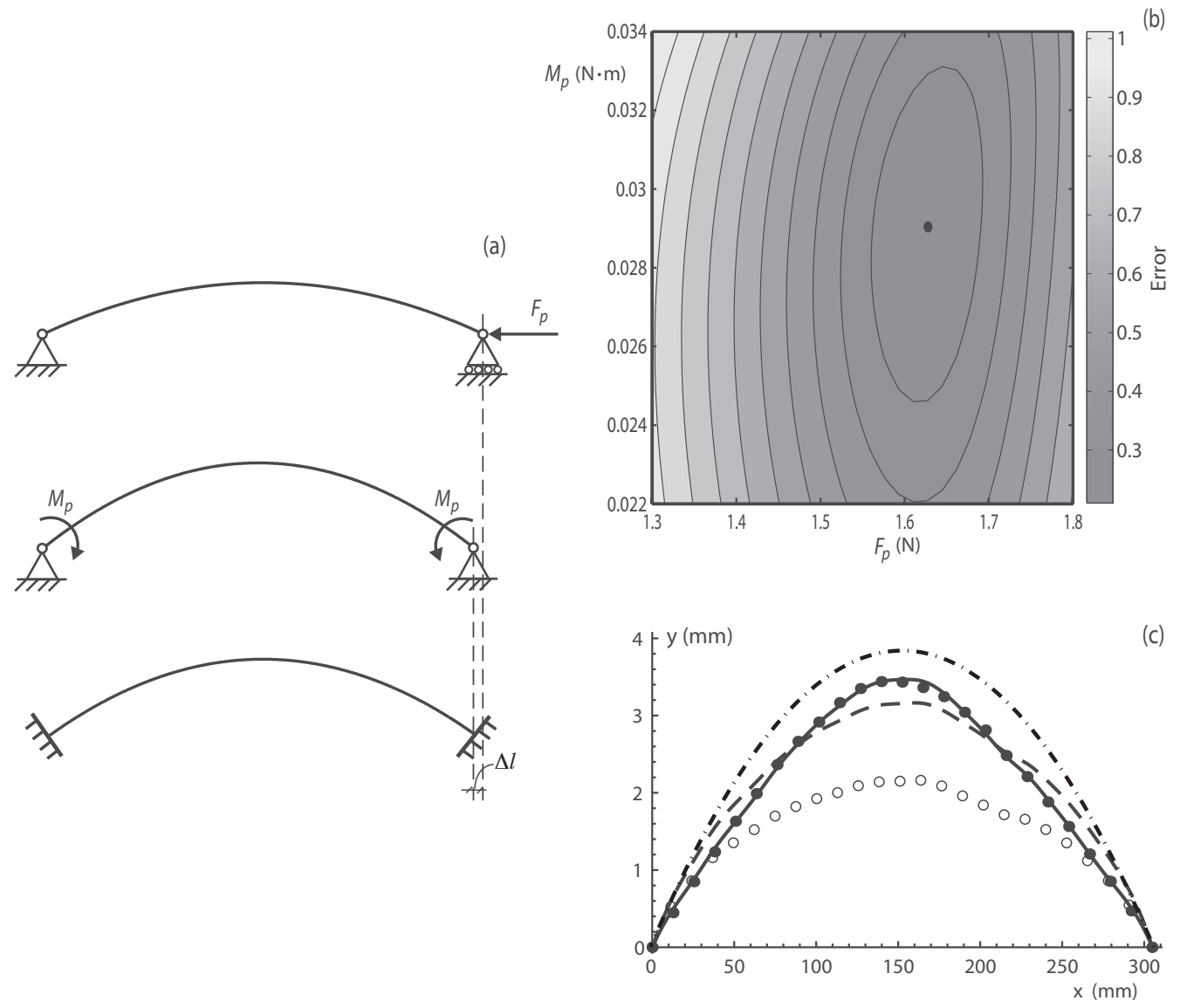

Figure 4: Illustration of prestressing procedure. (a) Schematic showing axial force and bending moment stages of prestressing. (b) Error norm vs. prestressing end force and end moments. (c) Design configuration (dashed-dotted line), measured pre-clamping configuration (empty circles), configuration after application of end force (dashed line, FEA) and end bending moments (solid line, FEA), and measured post-clamping configuration (filled circles). 
The final result (solid line) agrees very well with the experimentally measured configuration $y_{c}$ (filled circles). Note that the end displacement caused by $F_{p}$ in the first prestressing stage, shown in Figure 4 (a), shortened the arch by $\Delta l=4.59 \times 10^{-2} \mathrm{~mm}$. To maintain the total length of $304.8 \mathrm{~mm}$, it would therefore be necessary to begin with a slightly longer arch. However, given the small magnitude of shortening compared to the overall length, starting with a longer arch would produce negligible effects so this correction was not performed.

At this point in the modeling, the analyst has a choice of whether to use the prestressed FEA geometry or the experimentally measured clamped geometry (with the prestress forces transferred in). The experimentally measured configuration obviously better matches the true configuration; however, when transferred in, the prestresses will not be in equilibrium as they equilibrate in the (slightly different) FEA results. It was therefore decided to use the FEA result as the starting point for the subsequent static forcedisplacement analysis (and dynamic analysis later) as it closely matches $y_{c}$ and is in static equilibrium.

For a typical shallow arch, at certain levels of static loading, multiple equilibria (stable and unstable) exist, as shown in the load-deflection diagram in Figure 1 (b). For perfect structures, branching of the primary solution path may occur. Nevertheless, since the structure described in Section 2 is an imperfect structure with no branching, we instead have disconnected paths. Figure 5 shows both the experimental and FEA static equilibrium results for a point load applied at the midspan of the arch. Parts (a), (b), and (c) show the force magnitude plotted against the displacement measured at the left quarter point $\left(\mathrm{d}_{1}\right)$, midspan $\left(\mathrm{d}_{2}^{*}\right)$, and right quarter point $\left(\mathrm{d}_{3}\right)$, respectively. A practical issue arose in measuring the displacement at midspan due to the loading mechanism being placed coincident at this location which blocked the line of sight for the DIC cameras. The displacement in this region was therefore interpolated using an $8^{\text {th }}$ order polynomial using the known data to each side of the missing data. The excellent agreement between the experimental and numerical displacements at the left (Figure 5 (a)) and right (Figure 5 (c)) quarter points (which were directly measured by DIC) provides some level of confidence that the interpolated displacements at the midspan (Figure 5 (b)) are accurate. Since the test was performed using displacement control it was possible to follow the downward component of the equilibrium paths, which would not be possible with force control as these regions are unstable. However, the force was applied through single sided "pushing" con- 
tact, and thus the system did "jump" from point (iii) to (iv) in Figure 5 (b) (which is a region of compressive force).

Interestingly, although loaded at midspan, the arch snaps-through in an asymmetric configuration. This is apparent when comparing the two quarter point displacements in Figures 5 (a) and (c). The left quarter point in fact rises up slightly at first before eventually snapping-through, after which the structure returns to a symmetric configuration. This is made more obvious by the full field displacements shown in Figure 6 for the four equilibrium states labeled (i) through (iv) in Figure 5. The empty circles denote the interpolated (fitting an $8^{\text {th }}$ order polynomial to the filled circles) experimental results. The solid lines from the FEA results appear to faithfully represent the true geometry when compared with the experimental results.

The stability of the system may be investigated by determining the undamped natural frequencies for small oscillations about the equilibrium configurations. A stable system will exhibit positive real natural frequencies,
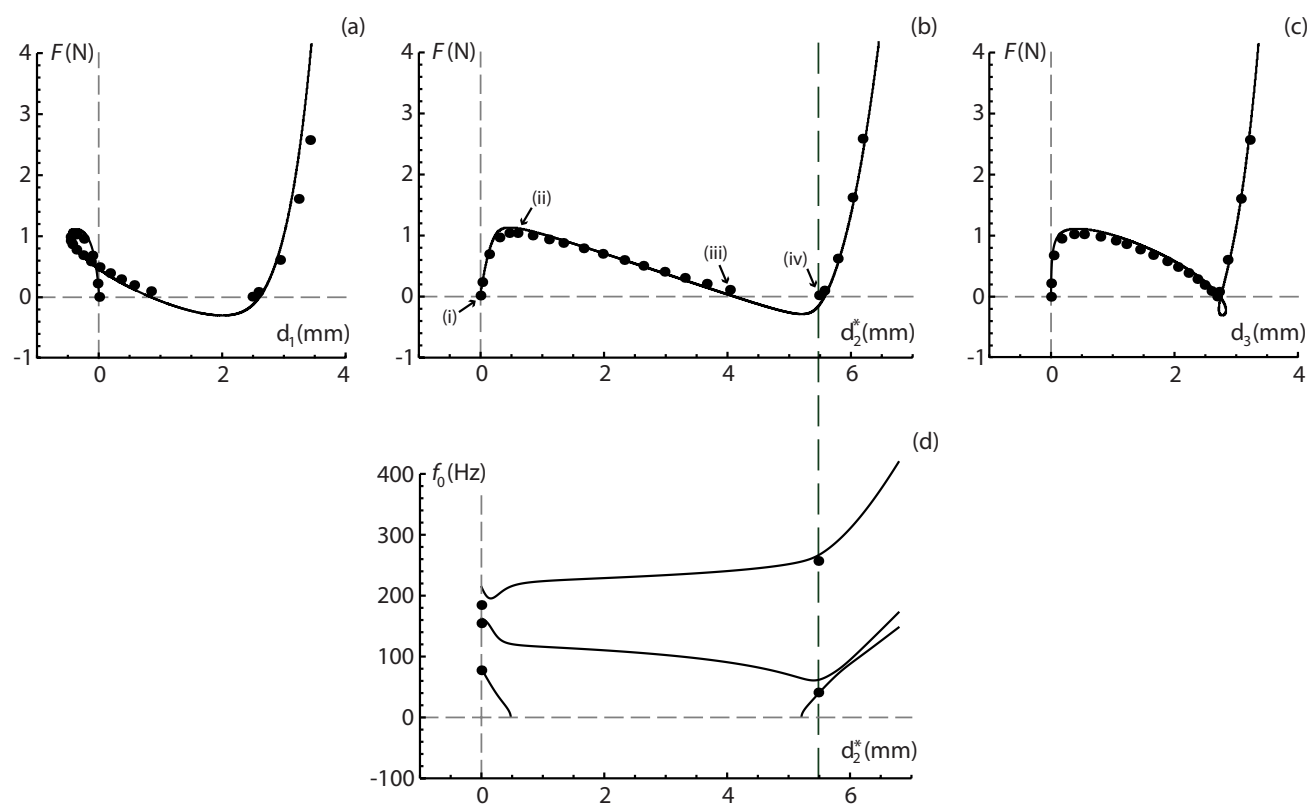

Figure 5: Comparison of experimental (filled circles) and FEA (solid lines) static forcedisplacement relationship for midspan point loading: displacement measured at (a) left quarter point, (b) midspan, and (c) right quarter point. (d) Comparison of experimental and FEA natural frequency vs. midspan displacement. * Indicates interpolated displacement measurement. 
(i.e., oscillatory response) for all modes about an equilibrium, whereas an unstable system will produce complex valued natural frequencies (i.e., hyperbolic divergence from an equilibrium). Figure 5 (d) shows the natural frequencies for the first three modes of the structure plotted against the midspan displacement of the equilibrium configuration. The experimental natural frequencies were obtained using an impact hammer and laser vibrometer measuring oscillations at the midspan. Experimental measurements could only be taken at the two unloaded stable equilibrium configurations (i) and (iv) (point (iii) is unstable), and were performed with the displacement control mechanism removed from contact. This was important because the displacement control mechanism produces what is essentially a pin constraint at its point of application, thoroughly changing the nature of the arch dynamics. Therefore it would not match the FEA results, which correspond to the ideal case of a non-inertial point load. The FEA results exhibit the characteristic frequency drop for the first mode, eventually dropping to zero at the limit point (which would be the bifurcation point in the perfect system as will be shown later) of the force-displacement relationship in Figure 5 (b) (this frequency is not plotted in the unstable regions as it is complex valued). The experimental natural frequencies exhibit excellent agreement with the FEA results at both the initial and snapped-through unloaded equilibrium configurations. In the snapped-through state, however, only a single

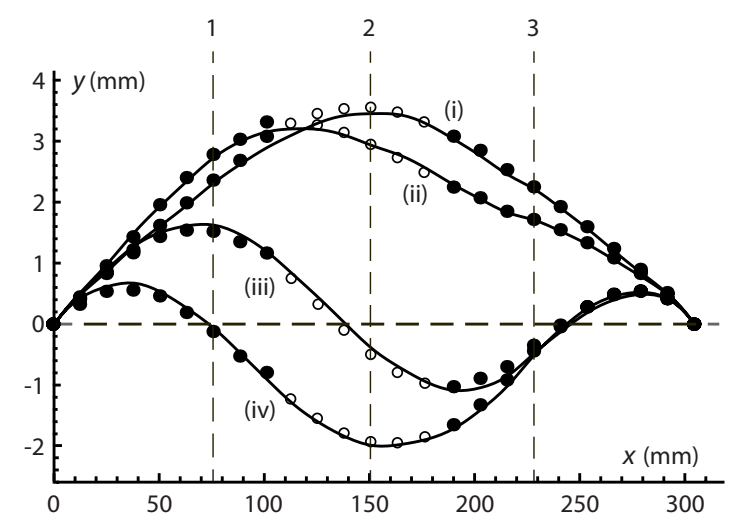

Figure 6: Comparison of experimental (filled circles for measured, empty circles for interpolated) and FEA (solid lines) arch configurations at the loads labeled (i) through (iv) in Figure $5(\mathrm{~b})$. The vertical dashed lines indicate the three locations at which $d_{1}, d_{2}^{*}$, and $d_{3}$ were measured. 
frequency below $100 \mathrm{~Hz}$ could be discerned from the experiments, while two frequencies are observed in the FEA results. This is not surprising when looking at how close the first and second mode frequencies are in this region in the FEA results.

The FEA was extended to the case of a uniform distributed load scenario. Due to the difficulty in performing a static experiment for a distributed loading, there is no experimental data for comparison. However, the similarity of the experimental data and FEA results for the point load scenario gives confidence in the FEA results. The static equilibrium path and the stability of the prestressed structure are presented in Figure 7 (solid lines). The static force-displacement for the distributed load is used as a guide to aid in defining snap-through in the dynamic response in Section 4. The load-displacement diagram for non-prestressed structure (dashed line) is also shown in Figure 7. We will revisit the influence of prestress on the snap-through boundaries in Section 4.

As previously mentioned, the arch used in the experiments had fabrication
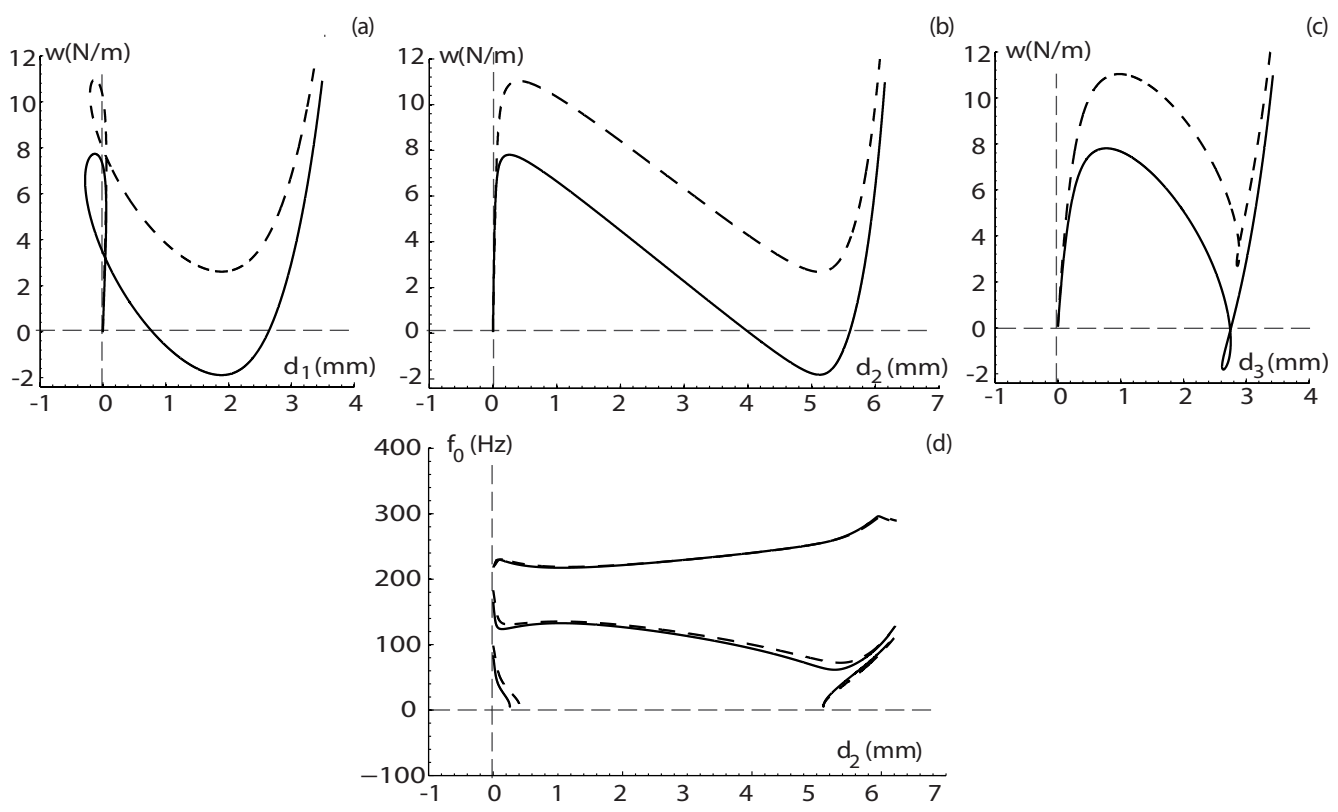

Figure 7: FEA static force-displacement relationship of prestressed structure (solid lines) and non-prestressed structure (dashed lines) for uniformly distributed loading: displacement measured at (a) left quarter point, (b) midspan, and (c) right quarter point. (d) natural frequency vs. midspan displacement. 
imperfections. For the design configuration (symmetric circular arch), the force-displacement diagram shows that the primary path corresponding to the symmetric configuration branches into an asymmetric configuration (Figure $8(\mathrm{a})$ ). The stability of the solution changes along the equilibrium path (as shown by the change in sign of the lowest natural frequency (squared) in Figure $8(\mathrm{~b})$ ). The lowest natural frequency (squared) becomes negative when the asymmetric solutions appear, then the second natural frequency (squared) becomes negative when the limit point is reached. The symmetric solution remains unstable until the natural frequencies (squared) become positive again in the snapped-through configuration. Since after the bifurcation point the solution is unstable under load control, the structure will jump to a remote configuration as this point is reached, making the bifurcation the true snap-through load as opposed to the more familiar limit point.
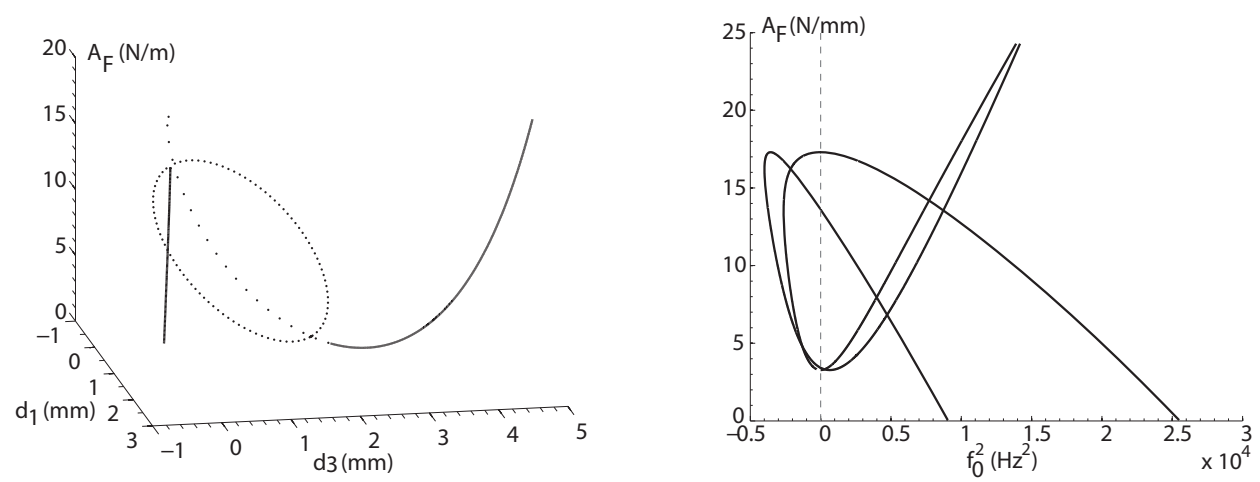

Figure 8: (a) Static equilibrium path and (b) the stability for the design configuration (circular arch) with distributed loading scenario. Dots in (a) represent unstable solutions.

\section{Dynamic Snap-Through}

In this section we discuss the transient behavior of a shallow clamped arch under harmonic distributed loading. Results obtained both experimentally and using numerical tools are compared. Different types of response are presented and important phenomena, such as chaos, are identified. Furthermore, we identify the boundaries that separate snap-through and non-snap-through regions in the parameter space, i.e., the snap-through boundary. The numerical simulations are then used to investigate the behavior of the arch further, e.g., we examine the influence of prestress on the snap-through boundary. 
Since snap-through is a highly nonlinear problem, numerical simulations of such phenomena require access to stable time-stepping schemes and in general to robust simulation environments. Several commonly used time stepping schemes encounter numerical difficulties when simulating such problems [15]. In this paper, the time-stepping scheme used is the $\alpha$ method $[16,17]$. For certain algorithmic parameters, the $\alpha$ method has the advantage of dissipating the non-physical high frequencies of the system and therefore helps the stability of the algorithm, without degrading the accuracy. The physical damping of the system is modeled using mass proportional damping, $C=\alpha_{0} M$. This assumption leads to the expression $\alpha_{0}=2 \zeta \omega_{0}$ for the modal damping of linearized oscillations about an equilibrium configuration. Experiments on the arch under investigation revealed that the first mode had a damping ratio of $\zeta=0.2 \%$ and a natural frequency of $75.5 \mathrm{~Hz}$ (see Figure $5(\mathrm{~d})$ ), which yields $\alpha_{0}=1.96$. The natural frequency, $f_{0}$, of the FEA model is $79.87 \mathrm{~Hz}$ yielding $\alpha_{0}=2.0$. This value was used in the numerical simulations and produced excellent agreement with experimental results. The results could likely be improved with a full proportional damping matrix, however, this was deemed unnecessary due to the already excellent agreement with a single damping parameter. Furthermore, the damping data point that was used was completely independent of the time series used in investigating snap-through which provides a level of confidence that the model is capturing the true physics naturally rather than being tuned to the measured results.

Two different arches were used in the dynamic experimental tests. Prior

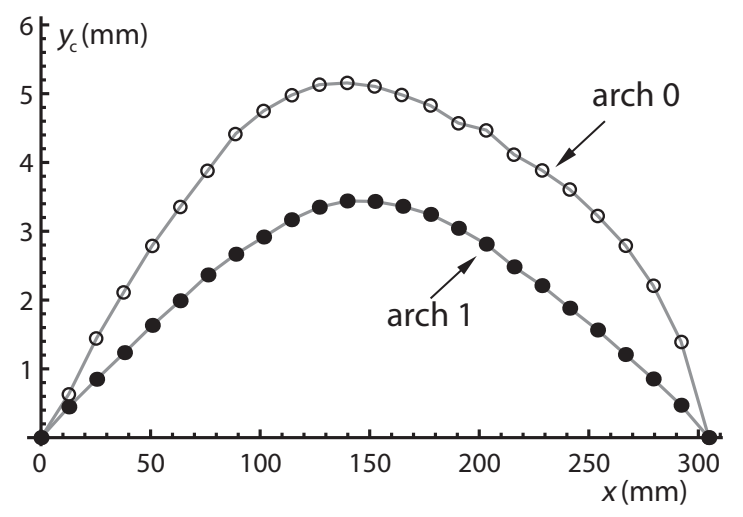

Figure 9: Measured post-clamping configuration of arch 1 (filled circles) and 0 (empty circles). 
to testing the arch for which the geometry is given in Section 2 (labeled as "arch 1"), a series of tests were performed on a different arch labeled "arch 0" (as it is a predecessor). The unloaded clamped configurations of arch 0 and arch 1 measured by DIC are shown in Figure 9. Both arches were fixed into the same clamps and therefore should have identical initial slopes at the supports. Inspection of the measured shape indicates that the right side of arch 0 has a much higher initial slope. This is likely an aliasing effect of having too coarse of a measurement grid and it is more probably that there is a "kink" in the arch near the support. Unfortunately, this arch was damaged (a sudden drastic change in both static and dynamic behavior was observed) during the course of large amplitude dynamic force testing. No FEA dynamics results or statics results will be introduced for arch 0 , however, some very interesting results were obtained prior to the damage that warrant discussion.

First, a forcing frequency sweep from $f_{F}=80$ to $200 \mathrm{~Hz}$ at a forcing
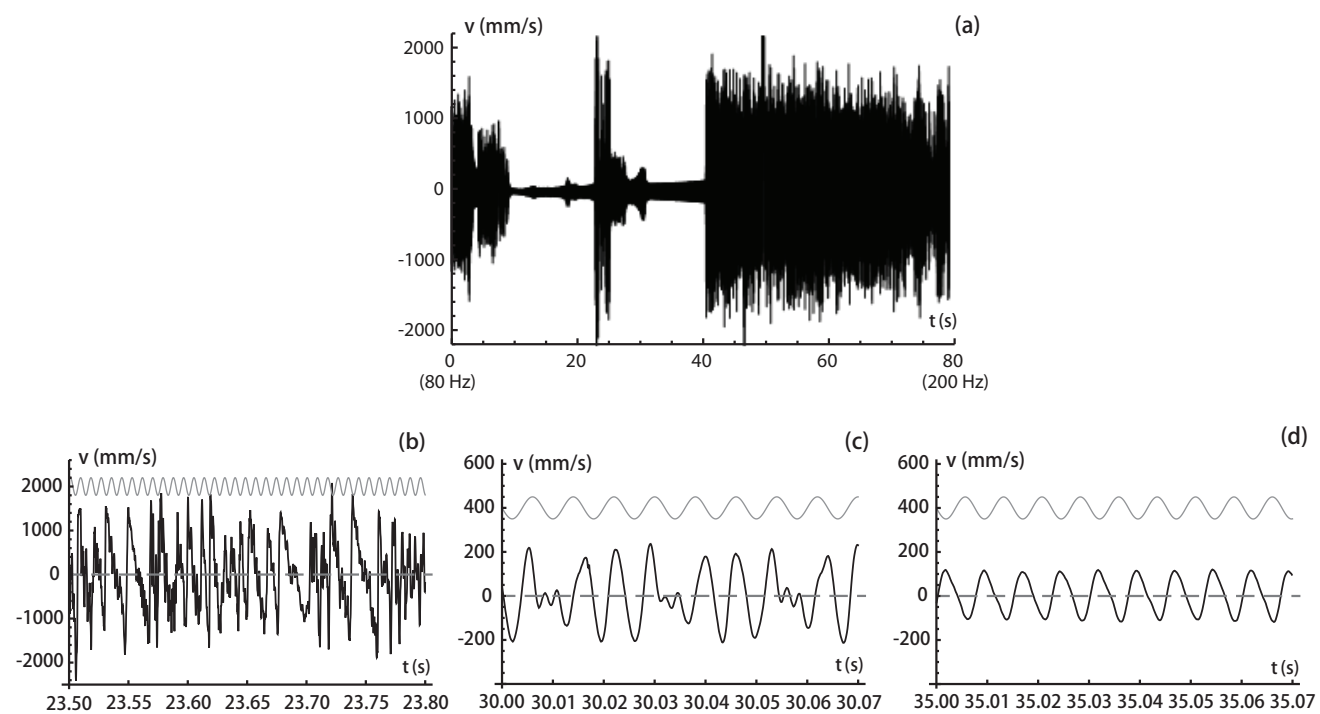

Figure 10: Experimental velocity (at midspan) time series under harmonic distributed forcing with $A_{F}=8.4 \mathrm{~N} / \mathrm{m}$ for arch 2: (a) frequency sweep from $f_{F}=80$ to $200 \mathrm{~Hz}$ over a period of 80 seconds exhibiting (b) chaotic, (c) period 3, and (d) period 1 response (note the three plots are over different time spans). The superimposed sinusoidal functions (gray) show the forcing frequency (their amplitude is not representative). 
amplitude of $A_{F}=8.4 \mathrm{~N} / \mathrm{m}$ over a period of 80 seconds was performed on arch 0 (Figure 10). The length of the time series exceeded the capability of the DIC for dynamic testing, and hence the response (midspan displacement) was measured with a laser vibrometer. The system exhibited many interesting types of response as shown in several zoomed in regions: Figure 10 (b) shows a chaotic response while Figure 10 (c) and (d) are period 3 and period 1 respectively. The period can be seen by counting the number of forcing cycles (forcing period may be interpreted from the superimposed harmonic function with a frequency equal to that of the forcing) necessary for the response to repeat.

While the amplitude of the velocity time series provides an indication of the energy of the response, one needs to examine the displacement directly to properly investigate snap-through. Figure 11 (a) shows a section of the displacement time series (from Figure 10 (b)) obtained by numerical

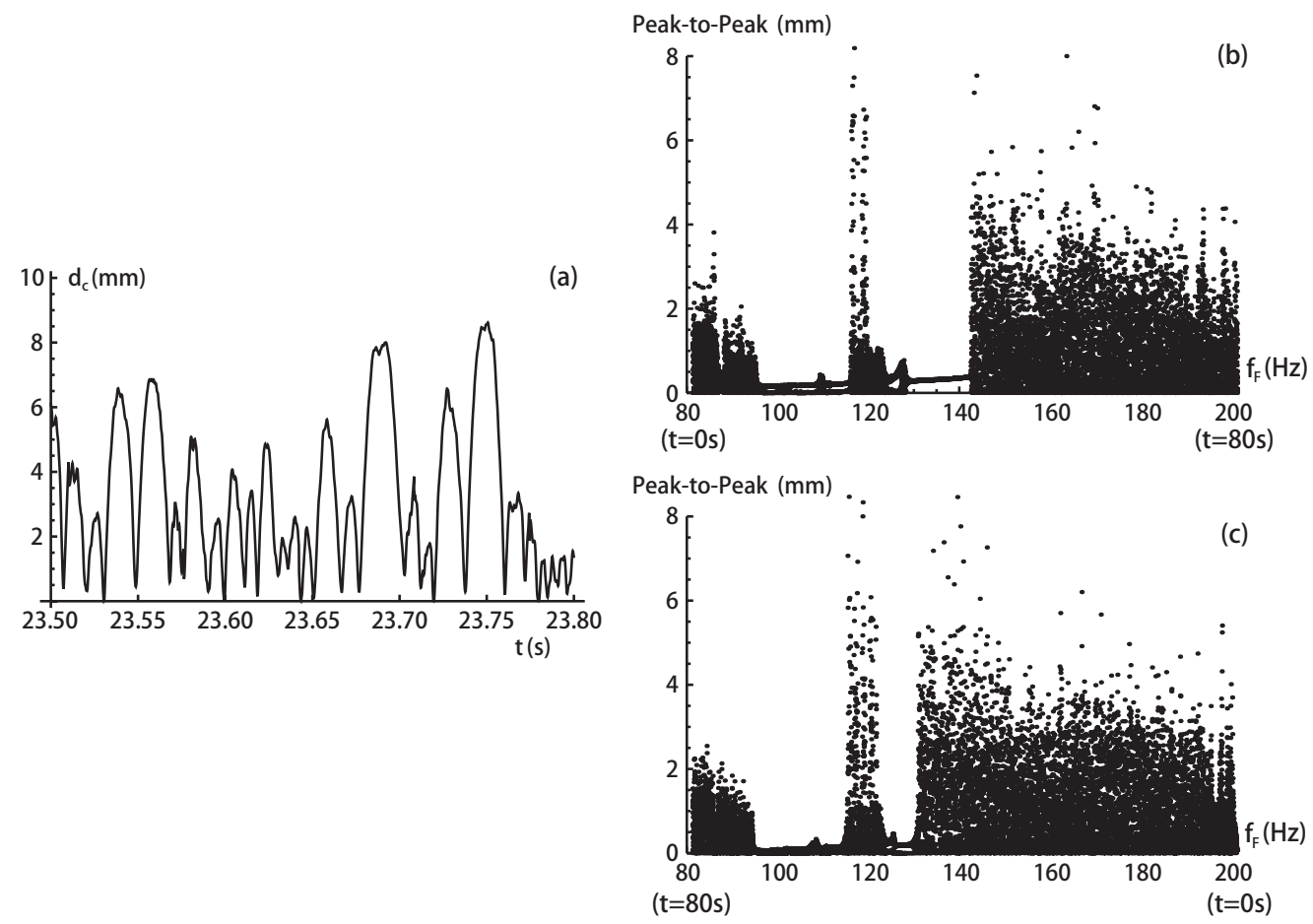

Figure 11: Experimental peak-to-peak amplitude bifurcation diagrams for arch 0: (a) time integration of velocity (see Figure 10 (b)), (b) sweep up (see Figure 10 (a)), and (c) sweep down bifurcation diagrams 
integration of the measured velocity. Due to a measurement bias (a slightly unsteady DC shift in the output) of the laser vibrometer, the absolute values of the displacements are unreliable. The peak-to-peak amplitude of the response still provides a useful insight into the bifurcations of the structure. The average measurement bias in Figure 11 (a) has been removed and the curve is shifted such that the minimum is zero for display purposes, however the true datum is unknown. Figures 11 (b) and (c) show the results of a peak-to-peak amplitude filter on the displacement time series (numerically integrated velocity) for a sweep up (Figure 10) and a sweep down. The filter simply plots the distance between each peak and the succeeding valley at the average frequency over that same interval (the frequency sweep rate was much slower than the typical response period, hence the forcing frequency was essentially constant for each response cycle). It is likely that some of the very small oscillations visible in the displacement time series are the result of noise, therefore many of the small values in Figures 11 (b) and (c) may not be true oscillations. However, the large amplitude oscillations are of primary interest and these are much less likely to be corrupted significantly by noise. Inspection of Figures 11 (b) and (c) shows a clear band of hysteresis from approximately 130 to $140 \mathrm{~Hz}$.

The bifurcation from small to large amplitude oscillations near $114 \mathrm{~Hz}$ is investigated in Figure 12 (a) and (b), which are obtained using an 80 second frequency up-sweep. The boundary is clearly quite complicated (possibly fractal) exhibiting several reversals between large and small oscillations. Sweeps over even narrower frequency bands could prove interesting and may
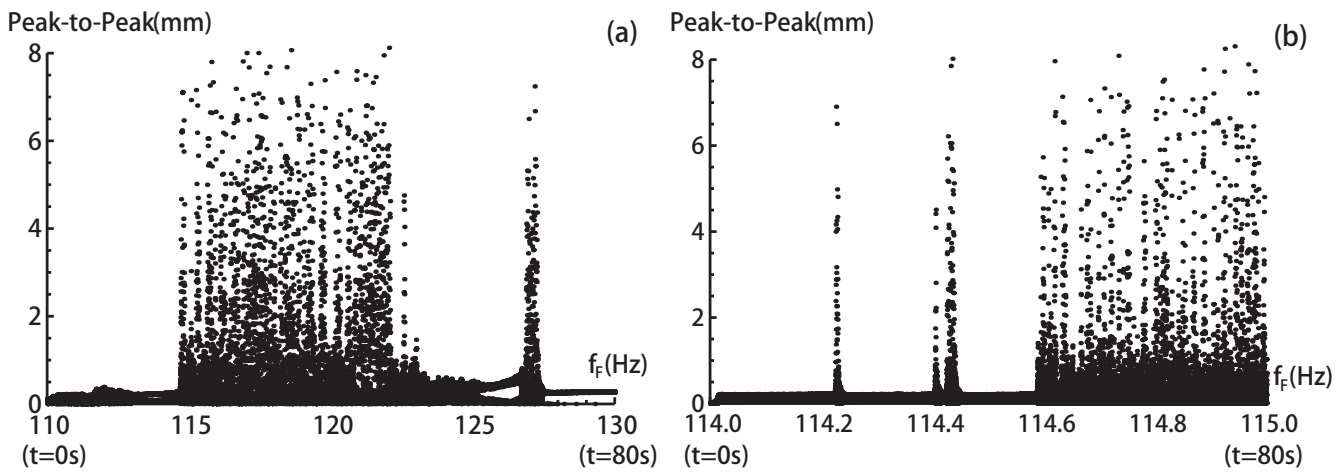

Figure 12: Experimental peak-to-peak amplitude bifurcation diagrams near $f_{F}=115 \mathrm{~Hz}$ bifurcation at two increasing levels of resolution. 
provide further evidence of a fractal boundary, however this would likely push beyond the abilities of the shaker to maintain a smooth frequency sweep rate over a narrow band.

The amplitude bifurcation diagrams may be used to develop snap-through rate diagrams. Plotting the snap-through rate provides a good indication of the temporal nature of the response, that is, whether it is transient, persistent but occasional, or frequent snap-through. Figure 13 shows a curve of the average snap-rate plotted against the forcing frequency. In order to determine the average snap-through rate, the number of snap-through events were counted in a 25 forcing period window bounding each frequency and multiplied by two in order to indicate both a snap-through and snap-back. The definition of dynamic snap-through is somewhat arbitrary. This is because, unlike in the static case, there is not necessarily a clear change in behavior at any point, the system may transit from single well oscillations to double well oscillations in a variety of ways and may snap-through in a transient or persistent fashion. It was decided therefore to define dynamic snap-through in the simplest fashion, that being when the peak-to-peak response exceeded a certain displacement threshold. Therefore the snap-through rate may be plotted for various definitions of the snap-through threshold as seen in Fig-

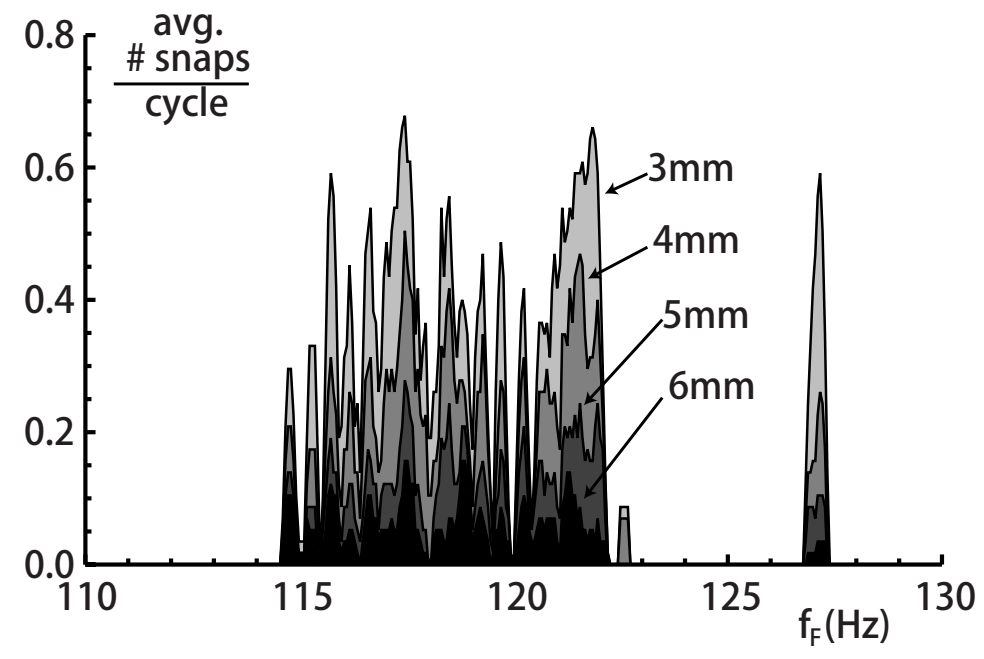

Figure 13: Experimental snap-through rate generated from Figure 12 using different definitions of snap-through. Lighter shading indicates lower snap-through definition. 
ure 13. For the choice of threshold values used, we observe similar ranges of frequencies that lead to snap-through responses, showing that there is a range of threshold values that can be used to define snap-through without compromising the identified snap-through region.

Returning to arch 1, a series of dynamic tests were performed on the arch under a harmonic distributed force. Figures 14 (a), (b), and (c) show the DIC measured displacement for $A_{F}=1.8 \mathrm{~N} / \mathrm{m}$ (arch 1 required much lower amplitudes to attain snap-through as it was somewhat more shallow) at 75 , 130, and $180 \mathrm{~Hz}$, respectively. Unfortunately, the dynamic DIC displacement measurements also contained a small bias in the displacement readings (a single unknown shift from the datum at $t=0$ ), hence the true datum $(d=0)$ may be shifted slightly from what is shown in Figure 14 (a) to (c). However, as in the previous discussion, the peak-to-peak displacements are of the primary concern. The biased nature of the response observed in the figure is expected, as the structure is very resistant to upward displacements, that is, the slope (stiffness) of the force-displacement relationship steepens sharply as the structure is raised upwards (negative displacements on the force-displacement plot shown in Figure 7).

Using the static equilibrium result for arch 1 (Figure 7 ) as a guide for
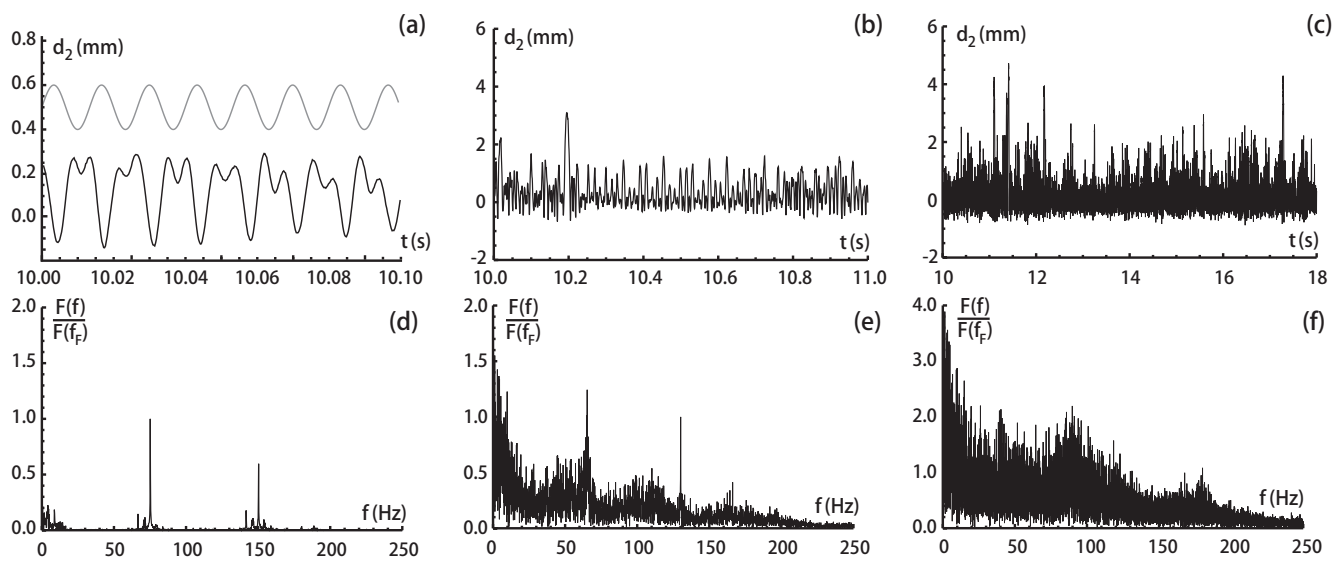

Figure 14: Experimental displacement (at midspan) time series under constant frequency harmonic forcing with $A_{F}=1.8 \mathrm{~N} / \mathrm{m}$ for arch 1: (a) $f_{F}=75 \mathrm{~Hz}$ (noisy harmonic nonsnap-through response), (b) $f_{F}=130 \mathrm{~Hz}$ (chaotic non-snap-through response ), and (c) $f_{F}=180 \mathrm{~Hz}$ (chaotic snap-through response). DFTs for: (d) $f_{F}=75 \mathrm{~Hz},(\mathrm{e}) f_{F}=130 \mathrm{~Hz}$, and (f) $f_{F}=180 \mathrm{~Hz}$. The superimposed sinusoidal function in (a) shows the forcing frequency (the amplitude is not representative). 
the selection of the snap-through threshold, the response can be categorized into snap-through and non-snap-through responses. Figure 7 shows that, at midspan, the separatrix (zero-load equilibrium position) separating the unsnapped from the snapped-through configuration is at approximately $4 \mathrm{~mm}$ when unloaded. Of course with a changing load, this separatrix is constantly shifting (not to mention the inertial forces, which add to the difficulty of relating static and dynamic snap-through). Nevertheless, since the mean forcing is zero this is the most justifiable snap-through definition. Using this value to define snap-through, Figures 14 (a) and (b) are considered as nonsnap-through responses while (c) is an example of a snap-through response.

The normalized (by the power at the forcing frequency $\left.F\left(f_{F}\right)\right)$ DFTs of the time series in Figure 14 (a), (b), and (c) are shown in Figure 14 (d), (e), and (f), respectively. The DFTs show a transition from a narrow band periodic to a broadband chaotic response. The determination of chaos through Lyapunov exponents $[18,19]$ for the experimental results is difficult to apply due to the dimensionality of the system. Therefore, chaos was determined by use of the method introduced in [20], which was shown to agree well with the more conventional Lyapunov exponents. In this method, the number of peaks above a certain magnitude ( $5 \%$ of the largest peak) on the DFT of the response is used as a metric to determine whether the response is chaotic or non-chaotic. The lower cut-off threshold is necessary to avoid counting noisy low power peaks near zero power, as even the DFT of a harmonic signal does not completely flatline away from the response frequencies. The method works because the DFTs of chaotic time series, which are typically wide band and very rough, exhibit many more peaks than DFTs of non-chaotic time series. The dichotomy in peak counts is typically so large that the determination of how many peaks necessary for a response to be considered chaotic is quite insensitive and easy to tune. Since quasi-periodic responses, like periodic responses, will exhibit only a handful of peaks, the method does not guarantee the identification of periodicity, only non-chaoticity. Responses for which more than $5 \%$ of the points on the DFT were peaks were considered to be chaotic. As previously mentioned, this selection was quite insensitive as the non-chaotic responses typically had less than $1 \%$ peaks, while chaotic responses were closer to $15 \%$ or higher. It is worth noting that, for a completely random sequence of data points, on average only $25 \%$ of the data points would be peaks, which makes this somewhat of an upper limit for the peak count. Using the peak-counting method, Figure 14 (a) can be considered as non-chaotic non-snap-through response. The harmonic forcing 
frequency, indicated by the superimposed curve in part (a), shows that the response, although somewhat noisy, does exhibit a primarily periodic nature. Figure 14 (b) is a chaotic but non-snap-through $(4 \mathrm{~mm})$ response, and Figure 14 (c) is chaotic with several snap-through events.

Numerical simulations performed for $A_{F}=1.8 \mathrm{~N} / \mathrm{m}$ obtain similar responses as those obtained experimentally. Figures 15 (a), (b), and (c) show the time series and (d), (e), and (f) show the DFTs for 71, 141, and $178 \mathrm{~Hz}$, respectively. The time series are plotted in true displacements, instead of peak-to-peak displacements, since, unlike experimental data, numerical results have no measurement bias. Using $4 \mathrm{~mm}$ as the definition for snapthrough and the peak counting method in [20] to determine the chaotic response, we identify Figure 15 (a) as a non-chaotic non-snap-through response, Figure 15 (b) as a chaotic non-snap-through response, and Figure 15 (c) as a chaotic snap-through response.

During the course of running the experiments, when the arch snappedthrough, it would occasionally settle and oscillate about the snapped-through configuration, referred to here as inverted snap-through. Occasionally it would snap back eventually, but often the arch had to be perturbed exter-
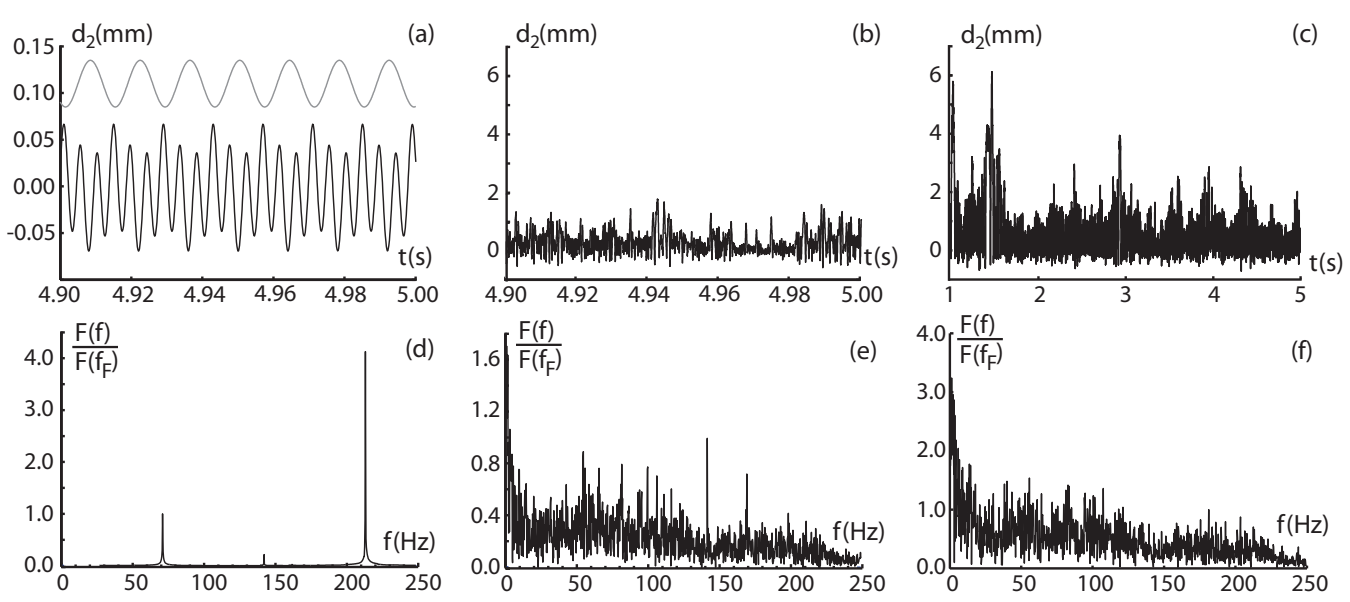

Figure 15: Numerical displacement (at midspan) time series under constant frequency harmonic forcing with $A_{F}=1.8 \mathrm{~N} / \mathrm{m}$ for arch 1: (a) $f_{F}=71 \mathrm{~Hz}$ (non-chaotic nonsnap-through response), (b) $f_{F}=141 \mathrm{~Hz}$ (chaotic non-snap-through response), and (c) $f_{F}=178 \mathrm{~Hz}$ (chaotic snap-through response). DFTs for: (d) $f_{F}=71 \mathrm{~Hz},(\mathrm{e}) f_{F}=141 \mathrm{~Hz}$, and (f) $f_{F}=178 \mathrm{~Hz}$. The superimposed sinusoidal function in (a) show the forcing frequency (the amplitude is not representative). 
nally for it to snap-back (Figure 16 (a)). Even though the response after the transient period does not contain any snap, all responses that presented this type of behavior are labeled as snap-through as in order to oscillate around the snapped-through configuration, the arch had to snap-through at least once. Since the arch is not designed to operate in its snapped-through state, oscillations around this configuration, although not as violent as persistent snap-through (the arch snaps-through and snaps-back continuously), are obviously undesirable. For the forcing parameters for which inverted snap-through occurred, there also typically existed persistent chaotic snapthrough (although it was often difficult to find). In experiments where the

(a)

(b)
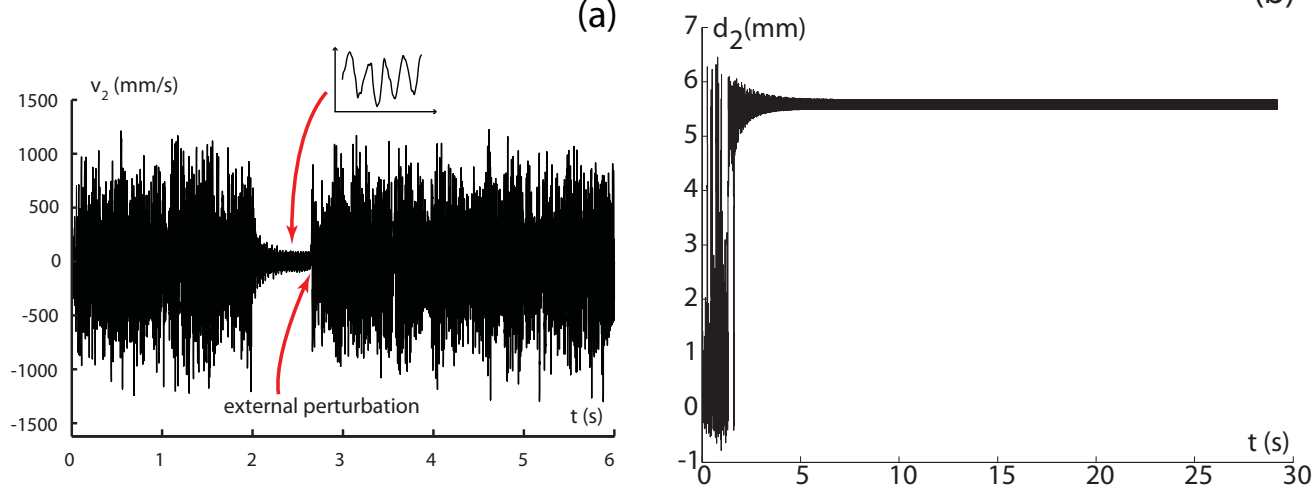

(c)
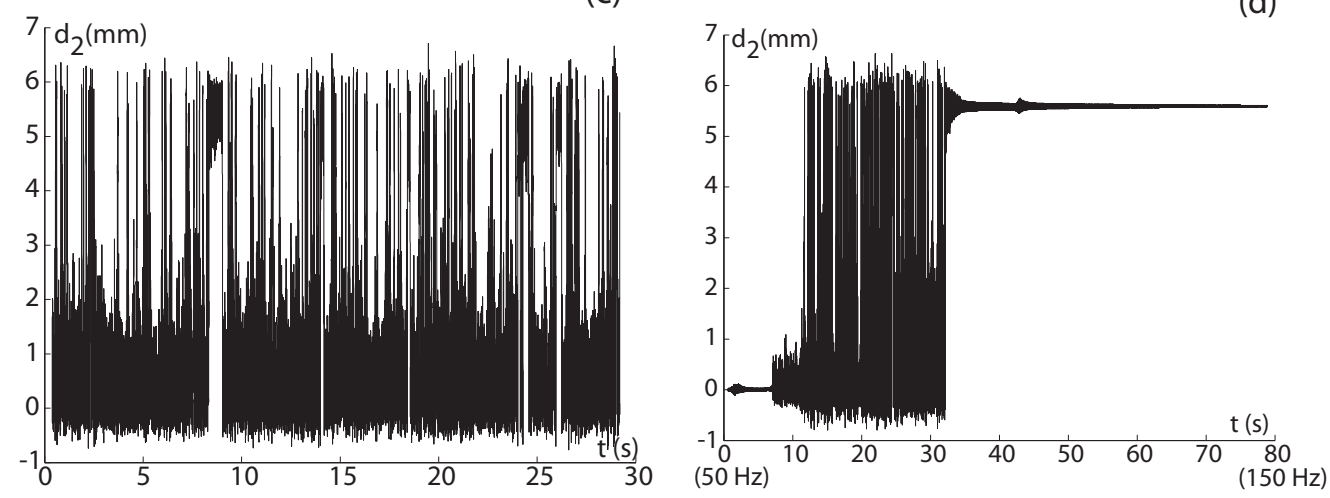

Figure 16: (a) Experimental time series showing inverted snap-through (getting "stuck" near the snapped-through configuration) response that needs to be removed with external perturbation, (b) inverted snap-through response obtained numerically, and (c) coexisting chaotic response obtained numerically, (d) inverted snap-through response under a frequency sweep that does not snap-back. 
arch would oscillate about the snapped-through configuration, the tests were re-run until there was a long enough period of persistent snap-through over which to determine the snap-through rate.

Similar behavior was also observed in numerical simulations. Figure 16 (b) shows a response obtained for $A_{F}=3.6 \mathrm{~N} / \mathrm{m}$ and $f_{F}=137 \mathrm{~Hz}$; the structure settles into oscillations around the remote configuration. When forcing with a different initial phase is applied, a co-existing chaotic persistent snapthrough response is identified (Figure $16(\mathrm{c})$ ). Once the arch settles at the snapped-through configuration, a force larger than the one causing it to snapthrough is required to push it to snap-back. Another example is shown in Figure $16(\mathrm{~d})$. This figure shows a result of a forcing frequency sweep from $f_{F}=50 \mathrm{~Hz}$ to $150 \mathrm{~Hz}$ at a forcing amplitude of $A_{F}=3.6 \mathrm{~N} / \mathrm{m}$ over a period of 80 seconds. At approximately $t=35$ seconds, the arch settles into oscillations around the snap-through configuration and even as we sweep through the frequency, the arch stays in that configuration and does not snap-back. Figure 16 (c) also serves to highlight the dichotomy in the response amplitudes exhibited by the system. The density of the time series shows that responses are typically either $<2 \mathrm{~mm}$ or much larger than $2 \mathrm{~mm}$, implying that dynamic snap-through is a distinct phenomena.

In order to characterize the snap-through behavior and determine the boundaries in the forcing parameter space $\left(f_{F}, A_{F}\right)$ separating the snapthrough from the non-snap-through domain, a series of 30 second tests were performed over a grid of forcing frequencies and amplitudes. To minimize extraneous data, only the midspan response was measured using a laser vibrometer. The midspan is the most obvious choice for the test point and provides sufficient data to characterize snap-through. This is supported by state (iii) in Figure 6 which shows that even for asymmetric snap-through the midspan deflection remains large. The measured velocities are integrated and the snap-through rate is determined in the same manner as previously discussed for arch 0 (4 $\mathrm{mm}$ is used as the threshold for snap-through). When a response exhibited a snap-through rate greater than zero, that is, exhibited any snapthrough events at all, it was considered as a snap-through response. The chaoticity of the response is determined through the peak-counting method. The resulting snap-through boundary is shown in Figure 17 (a). The responses are categorized into three groups: (1) snap-through (filled circles), (2) chaotic non-snap-through (empty circles), and (3) non-chaotic non-snapthrough (small dots). The intersections of the dashed gray lines are the forcing parameters of the three responses shown in Figure 14. 
The persistent snap-through responses obtained experimentally were also all chaotic, as opposed to the behavior observed in the SDOF arch studied

(a)

(b)
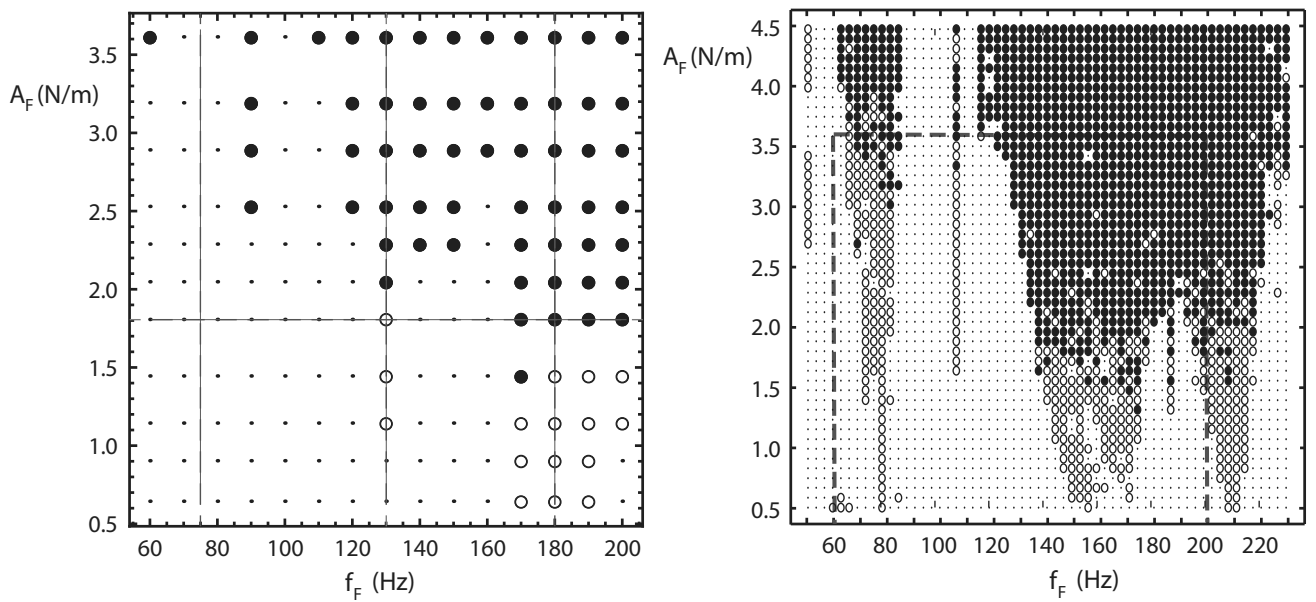

(c)

(d)
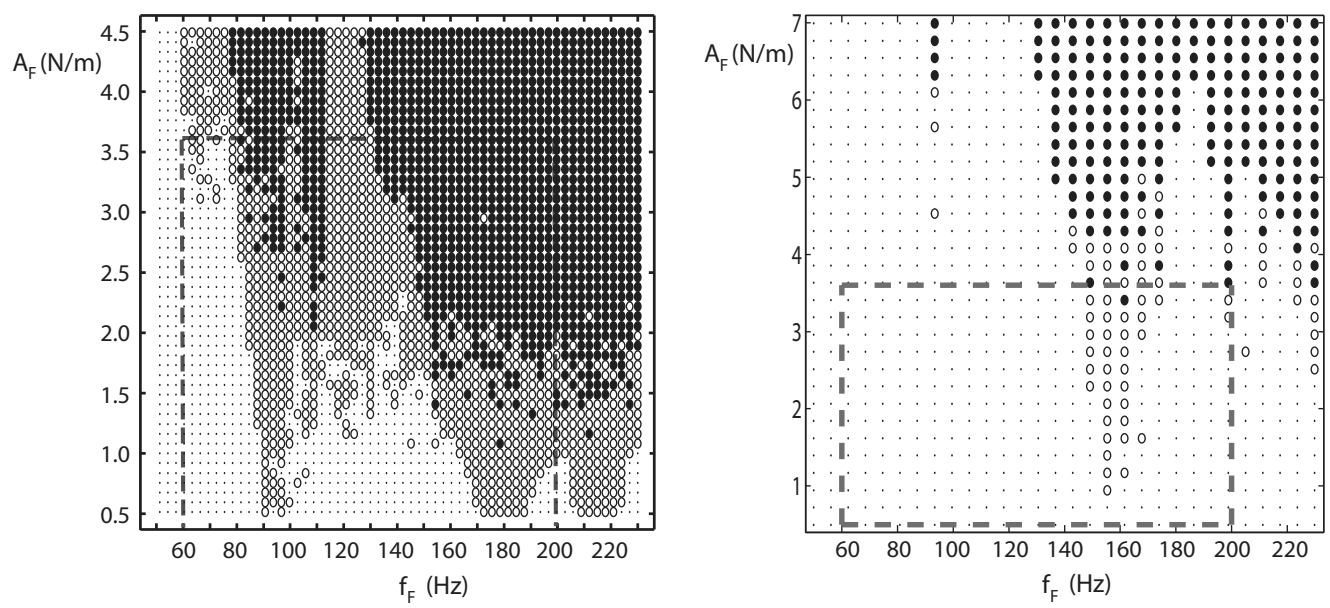

Figure 17: Snap-through and chaos boundaries in forcing amplitude and frequency space obtained through: (a) experiment and (b) numerical simulations for the measured clamped configuration with prestress, (c) the measured clamped configuration without prestress, and (d) the design configuration. The responses identified are snap-through (filled circles), chaotic non-snap-through (empty circles), and non-chaotic non-snap-through (small dots). The intersections of the dashed gray lines in (a) denote the locations of the three responses shown in Figure 14. The dashed boxes in (b), (c), and (d) indicate the ranges of forcing parameters used in (a). 
in [10]. In that study, it was found that non-chaotic large oscillation snapthrough events were quite common, while chaotic non-snap-through events were rare. It is expected that the non-chaotic persistent snap-through oscillations, although not observed in tests on arch 1, would be found for very large amplitude forcing where the arch would be obliged to snap-through in phase with the forcing. Nevertheless, based on tests on arch 0 , the loads were kept low in the experiments to avoid damaging arch 1 leaving the phenomena to be investigated by numerical means only. For the counteracting case of chaotic non-snap-through response, it is not surprising that a continuous arch, an infinite dimensional system, is more apt to chaotic motion than a SDOF model.

The snap-through boundary was also determined through numerical simulations (Figure 17 (b)). The dashed box shown in the figure indicates the ranges of forcing parameters used in the experiments. For each pair of forcing parameters, the structure is excited from its equilibrium configuration and the simulation is performed for 6 seconds. Only the last two seconds of the simulations (eliminating the transient) are used to determine whether the response snaps-through and whether it is chaotic. If the response passes the threshold value $(4 \mathrm{~mm})$, it is considered as a snap-through response. The chaotic response is identified using the same method as for the experimental data. Similar to the experimental results, snap-through (filled circles), chaotic non-snap-through (empty circles), and non-chaotic non-snap-through (small dots) responses are identified.

The numerical and experimental snap-through boundaries show similar features. First, we observe two regions of frequencies that do not show snapthrough in between frequencies that do. In the experimental data, the regions are approximately at $f_{F}=75 \mathrm{~Hz}$ and $f_{F}=100 \mathrm{~Hz}$. In the numerical snapthrough boundary, they are approximately at $f_{F}=60 \mathrm{~Hz}$ and $f_{F}=100 \mathrm{~Hz}$. Second, the lowest forcing amplitude that causes snap-through is approximately at $A_{F}=1.4 \mathrm{~N} / \mathrm{m}$, and occurs around $f_{F}=170 \mathrm{~Hz}$ in both snapthrough boundaries.

In approximate analytical methods such as perturbation, multiple scales, and harmonic balance, often times one is able to observe interesting internal and combination resonances at frequencies commensurate to groups of natural frequencies or equal to their difference or summation [21]. As the nonlinearity grows these interactions grow increasingly more complex, while at the same time, the approximate analytical approaches used to study them become less accurate, until what is left is a continuum of behavior (or co- 
existing behaviors) that is very difficult to decompose into any sort of coherent structure. This is true even for low order discrete systems where structures such as nonlinear normal modes quickly grow very complex [22], a behavior which is magnified with the high dimensionality of FEM models of distributed systems. However, the primary, internal, and combination resonances may prove useful in predicting initial loss of stability of small (safe) response. For example the linear natural frequency of the first three unloaded modes (Figure $5(\mathrm{~d})$ ) are apparent as dips in the snap-through boundary in Figure 17(b), in what could be called primary resonance. The complex behavior near $160 \mathrm{~Hz}$, however, may hide internal resonance of the first and second modes, as the first mode is approximately commensurate with this frequency. The dip at $105 \mathrm{~Hz}$, is not immediately recognizable as a primary, combination, or internal resonance, however one must be careful when projecting linear natural frequencies up to higher energy levels (e.g., stiffening and softening springs).

In the snap-through regions of Figure 17 (a) and (b), inverted and persistent snap-through are not distinguished. This is because, as already discussed, they frequently coexist (see Figure 16 (a) for experimental, (b) and (c) for numerical). They are both detrimental in real structures, and both always involve at least a single snap-through event.

Numerical studies were also performed on the non-prestressed structure and on the design configuration. The snap-through and chaos boundaries for the non-prestressed structure (Figure 17 (c)) show that the region of chaotic non-snap-through is much larger than for the prestress configuration. Furthermore, as opposed to the snap-through boundary with prestress, for the non-prestressed structure, no inverted snap-through response was observed. This is likely a result of the non-existence of a stable unloaded static snapped-through configuration when no prestress in included. This is shown in Figure 7, where only a single root exists in the non-prestressed case. The snap-through region is also slightly shifted to higher frequencies compared to the prestressed structure.

The snap-through and chaos boundaries for the design configuration are shown in Figure $17(\mathrm{~d})$. This figure shows that the parameter space used in the experiments (dashed box) is almost outside of the region of snap-through when the design configuration is used (which corresponds to a deeper arch). The snap-through boundary, however, is somewhat similar in shape to the one obtained experimentally, even though it is shifted in both amplitude and frequency. Therefore, care should be taken when numerical tools are 
used to analyze the instability behavior of a real structure. Imperfections in fabrication, clamping force, and many other factors play a role in the transient behavior of a structure. This has many implications in engineering and design as it shows that structures such as shallow arches are exceptionally sensitive to boundary conditions and prestressing (which may be caused by thermal effects) and require careful investigation.

The contour plot of the rate of snap-through for Figure 17 (b) is presented in Figure 18 (a). The rate of snap-through is computed by averaging the number of snap-through events that occur in 100 forcing cycles after $t=4$ seconds. We observe that the average number of snap-through events is higher for low forcing frequencies. The change in snap-through boundary over length of simulation time is also of interest. From Figure 18 (b) we observe that the first snap-through occurs mostly within the first two seconds of the simulations. Therefore, longer simulation time is not likely to change the snap-through boundary significantly.

We also examine how the different threshold values used the as snapthrough definition influence the snap-through region (Figures 19 (a) and (b)). Figure 19 (a) shows snap-through boundaries for two threshold values: $3 \mathrm{~mm}$ (gray) and $6 \mathrm{~mm}$ (black) for the prestress structure. The boundaries are almost on top of each other showing that the separatrix (the unstable equilibrium separating the initial and snapped-through configurations) is a reasonable choice for the snap-through threshold. The change in the area of the snap-through region (normalized by the total area of the plot) for the prestressed structure obtained with different threshold values is shown
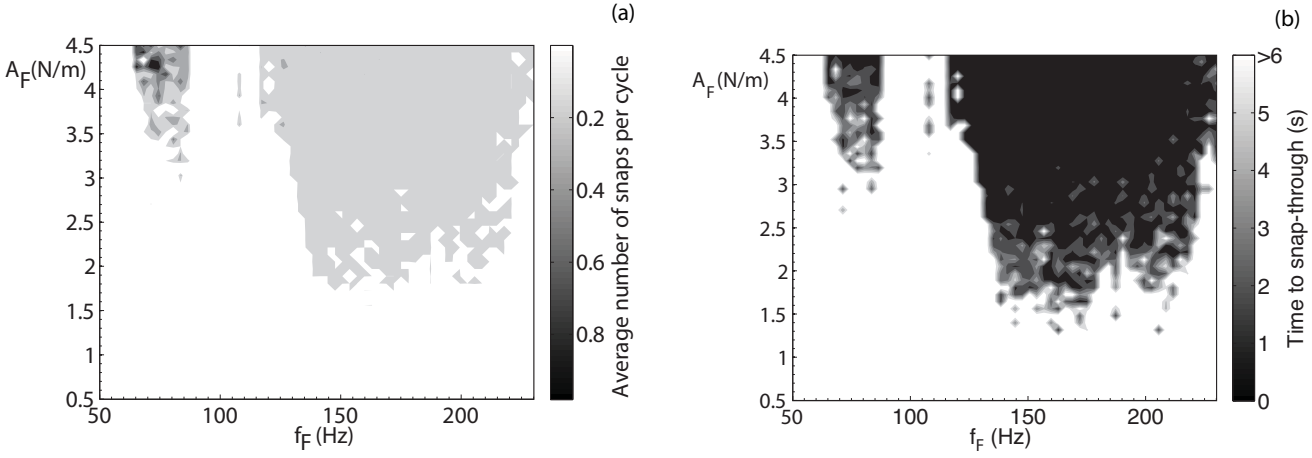

Figure 18: Contour plots for (a) snap-through rate and (b) time required to experience the first snap-through for the prestress structure. 
in Figure 19 (b). The snap-through area decreases rapidly between 0 and $0.5 \mathrm{~mm}$ and between $6 \mathrm{~mm}$ and $7 \mathrm{~mm}$. It decreases at a much slower rate between 0.5 and $3 \mathrm{~mm}$. The curve between 3 and $6 \mathrm{~mm}$ is almost flat showing a region where the determination of the snap-through region is not sensitive to the choice of threshold value, which reinforces the assertion that dynamic snap-through is distinct in that the dynamic response is either small or large amplitude. The plot of the snap-through area for the non-prestressed structure (Figure 19 (c)) shows a similar rapid decrease between 0 and $0.5 \mathrm{~mm}$ and between $6 \mathrm{~mm}$ and $7 \mathrm{~mm}$. No flat region is observed; the area decreases

(a)

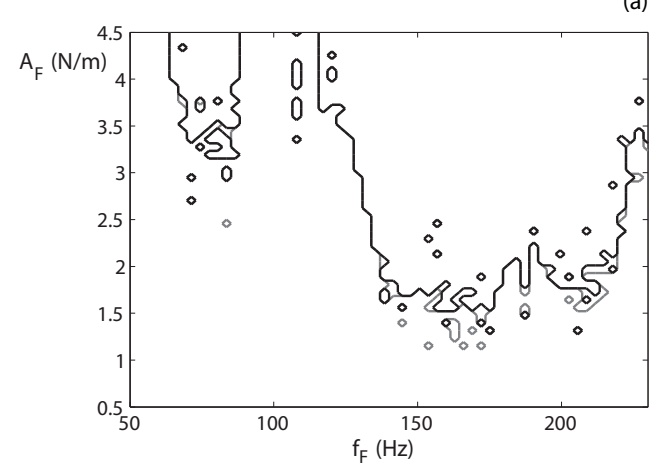

(c)

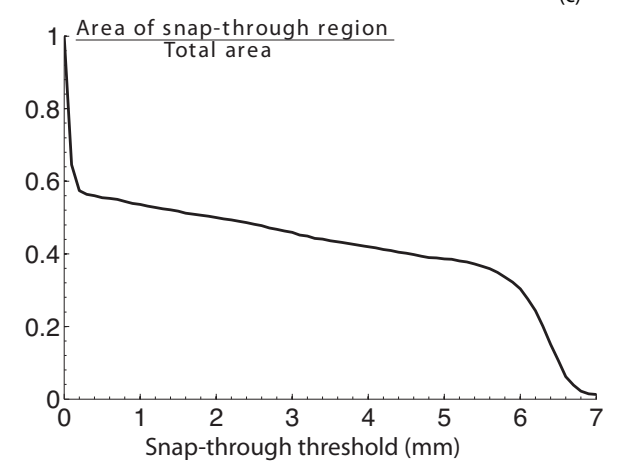

(b)

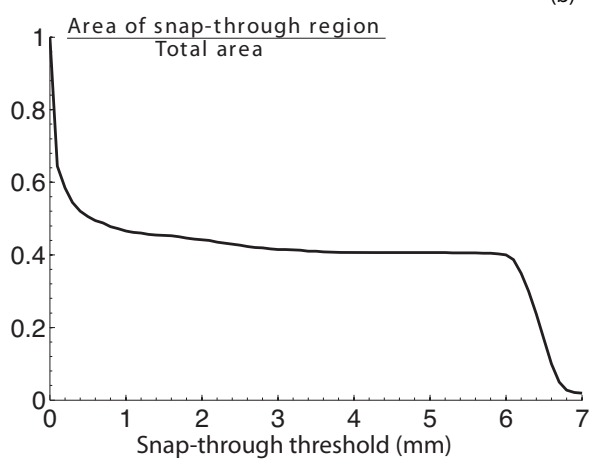

(d)

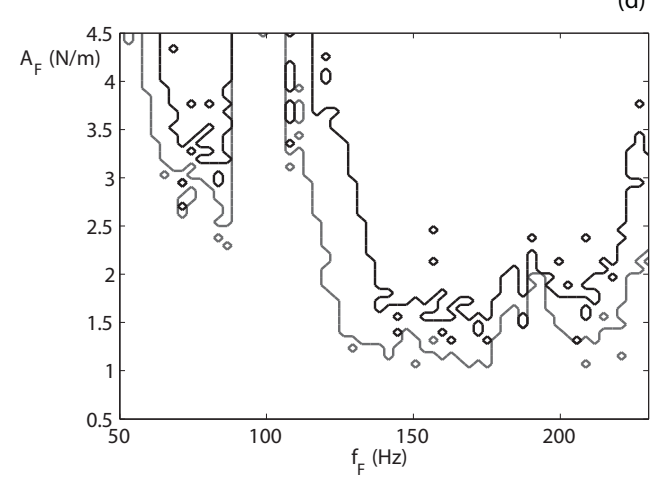

Figure 19: (a) Snap-through boundaries obtained using different threshold values: $3 \mathrm{~mm}$ (gray) and $6 \mathrm{~mm}$ (black). Area of snap-through region normalized by the total area for different threshold values for: (b) prestressed structure, (c) non-prestressed structure. (d) Snap-through boundaries obtained using different initial condition (black line indicates the boundary when the arch is excited from equilibrium configuration and gray line indicates boundary obtained when other initial conditions are used). Different initial conditions used expand the snap-through boundary. 
gradually between $0.5 \mathrm{~mm}$ and $6 \mathrm{~mm}$. We still can, however, argue that the value around the separatrix can be used as the threshold value without affecting the accuracy significantly since the variation of the snap-through area around the separatrix is small.

The use of different initial conditions leads to a more noticeable change in the snap-boundary, as shown in Figure 19 (d). The black line shows the boundary when the structure is excited from the at rest equilibrium configuration. The gray line includes additional forcing parameters that lead to snap-through when the structure is excited from other initial conditions (twenty initial conditions are used). If more initial conditions are used, the region where snap-through is possible is likely to expand more. Inside this region, a region where snap-through is inevitable, i.e., where all initial conditions lead to snap-through could also be identified.

In order to examine the arch behavior further, a series of simulations are performed for $A_{F}=3.6 \mathrm{~N} / \mathrm{m}$ with frequencies ranging from $60 \mathrm{~Hz}$ to $160 \mathrm{~Hz}$. Each simulation is performed for 6000 forcing cycles or more and the Poincaré points of the last 1000 forcing cycles (at a given forcing phase) are shown in the bifurcation diagram in Figure 20. A rich behavior is observed; we identify periodic, quasi-periodic, and chaotic responses. For the non-snap-through case, periodic (Figure 21 (a)), quasi-periodic (Figure 22 (a)) and chaotic (Figure 23 (a)) responses are identified. Both periodic (Figure 21 (b)) and quasi-periodic (Figure 22 (b)) responses are observed in the inverted snap-

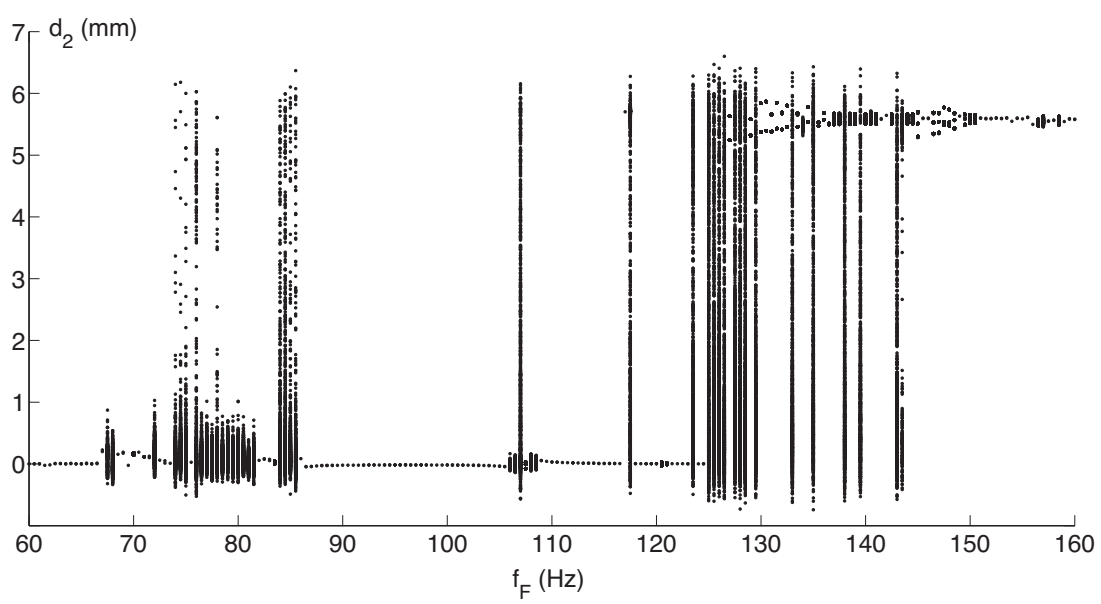

Figure 20: Bifurcation diagram for $A_{F}=3.6 \mathrm{~N} / \mathrm{m}$. 
through case. In the persistent snap-through case, we only observe chaotic responses (Figure 23 (b)).

For the range of forcing amplitudes used to determine the snap-through boundary in Figure 17 (b), we do not identify cases of periodic or quasiperiodic persistent snap-through responses. However, when larger forcing amplitudes are used, non chaotic persistent snap-through (Figure 24) are observed. The time series and the phase plot with the Poincaré sections obtained for $A_{F}=22 \mathrm{~N} / \mathrm{m}, f_{F}=120 \mathrm{~Hz}$ (Figure 24 (a) and (c)) show a period one persistent snap-through behavior. The time series plot for $A_{F}=34 \mathrm{~N} / \mathrm{m}, f_{F}=120 \mathrm{~Hz}$ is shown in Figure $24(\mathrm{~b})$ and the plot of the Poincaré points (Figure $24(\mathrm{~d})$ ) shows it is a quasi-periodic response.

(a)

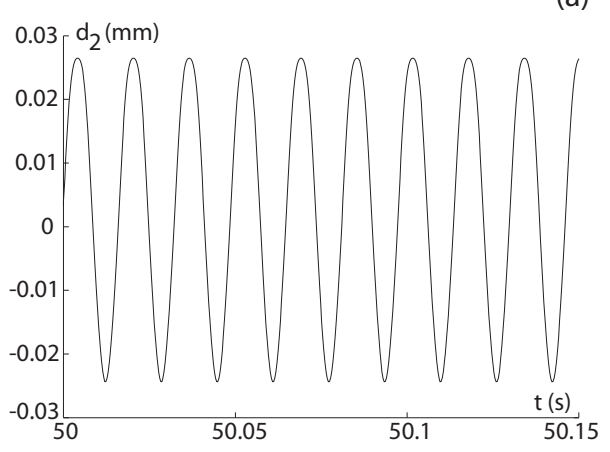

(c)

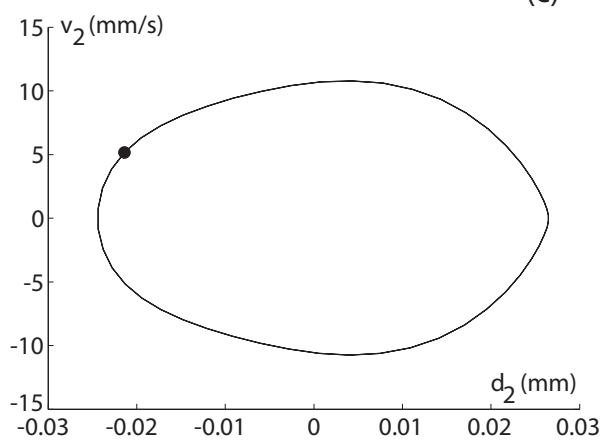

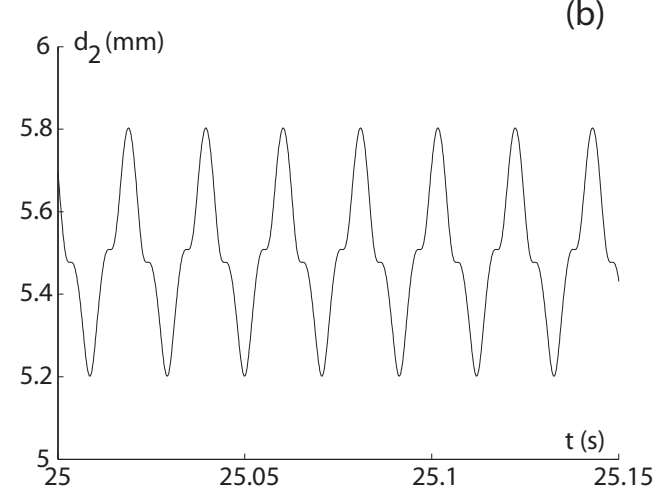

(d)

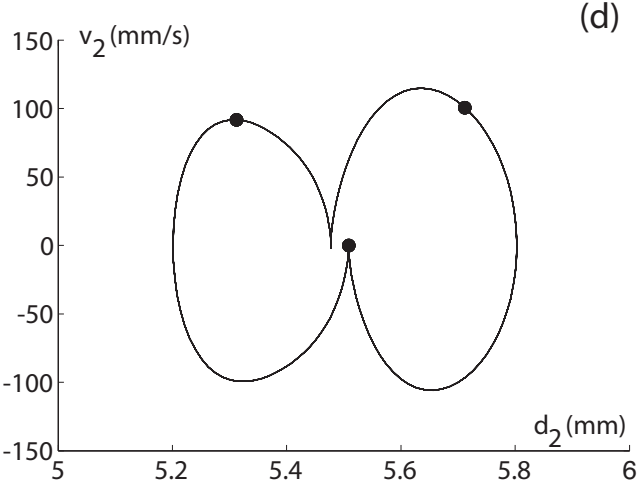

Figure 21: Time series for periodic responses: (a) no snap-through $\left(f_{F}=61.5 \mathrm{~Hz}\right),(\mathrm{b})$ inverted snap-through $\left(f_{F}=137 \mathrm{~Hz}\right)$. Phase plots and Poincaré points for $(\mathrm{c})$ no snapthrough, (d) inverted snap-through. 
(a)

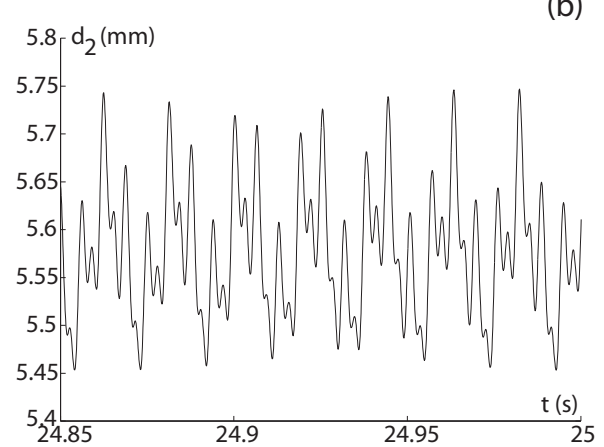

(c)
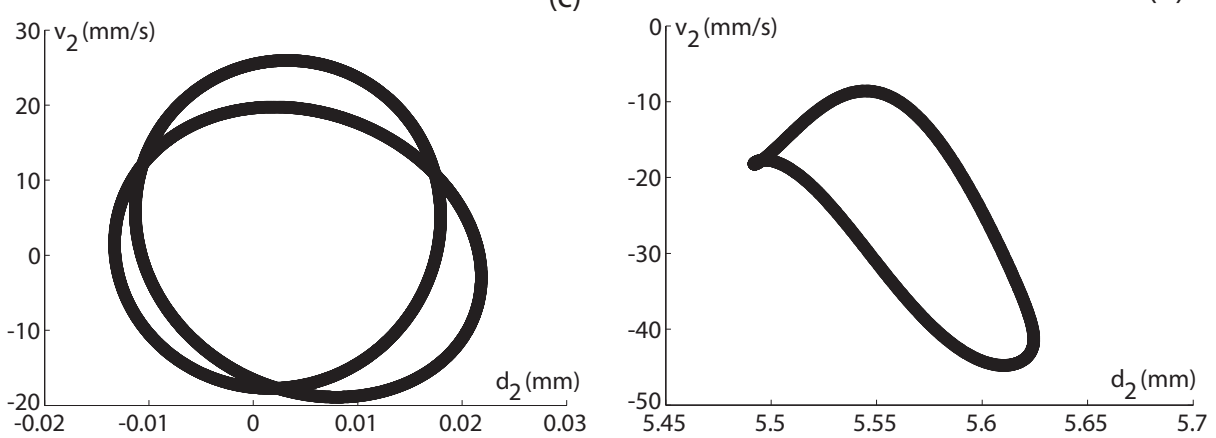

Figure 22: Time series for quasi-periodic responses: (a) no-snap-through $\left(f_{F}=120.5 \mathrm{~Hz}\right)$, (b) inverted snap-through $\left(f_{F}=158.5 \mathrm{~Hz}\right)$. Poincaré points for $(\mathrm{c})$ no-snap-through, $(\mathrm{d})$ inverted snap-through.

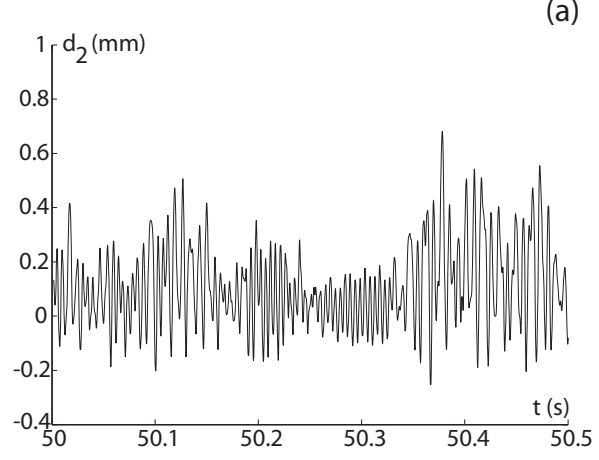

(a)

(b)

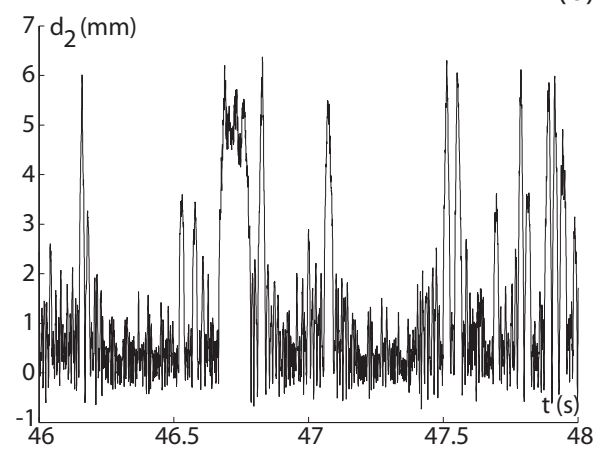

Figure 23: Time series for chaotic responses: (a) no-snap-through $\left(f_{F}=67.5 \mathrm{~Hz}\right)$, (b) persistent snap-through $\left(f_{F}=143 \mathrm{~Hz}\right)$. 
(a)

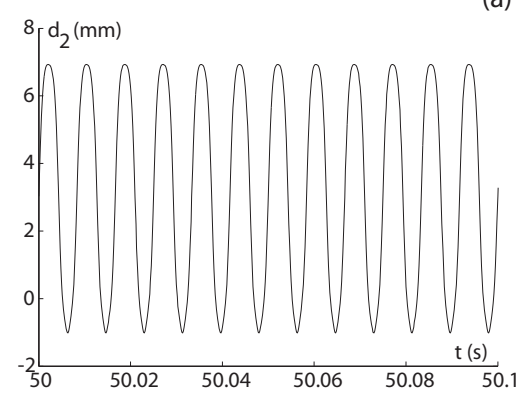

(c)

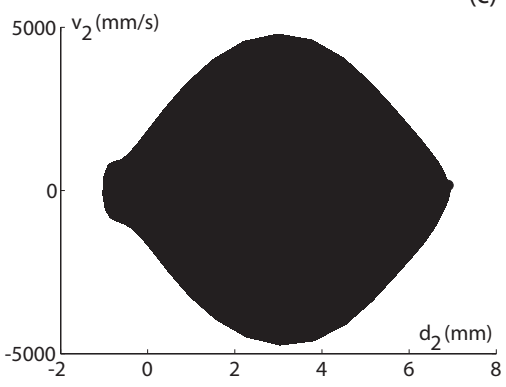

(b)

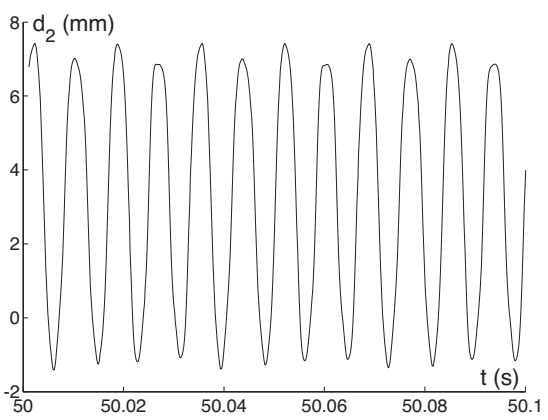

(d)

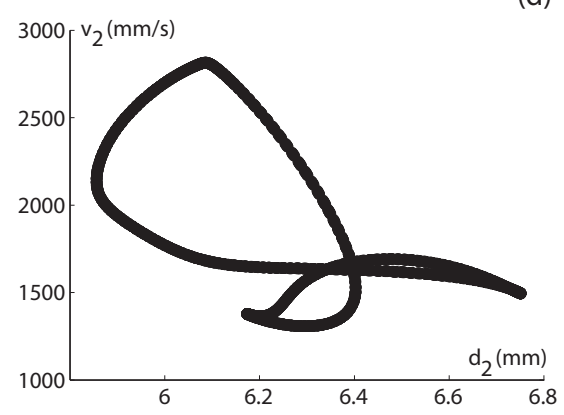

Figure 24: Time series for persistent non-chaotic snap-through responses: (a) periodic $\left(A_{F}=22 \mathrm{~N} / \mathrm{m} f_{F}=120 \mathrm{~Hz}\right)$, (b) quasi-periodic $\left(A_{F}=34 \mathrm{~N} / \mathrm{m} f_{F}=120 \mathrm{~Hz}\right)$. (c) Phase plot and Poincaré point that corresponds to (a), (d) Poincaré points that correspond to (b).

\section{Conclusions}

In this paper, a combined experimental - computational framework is used to analyze the transient behavior of clamped-clamped shallow arches. To ensure consistency with the experimental measurements, prestress is first applied in the FEA model and then the prestressed FEA model is used as the initial configuration for the static and dynamic simulations. The comparison of the measured and the simulated static load-displacement diagrams obtained under point load shows a good match. The FEA model is then used to obtain the static load-displacement diagram for the arch under distributed loading.

The study is then extended numerically to examine the response of the arch under harmonic distributed loading to determine regions in the forcing parameter space that lead to snap-through and chaotic response using the 
framework presented in [11]. The definition of snap-through is determined as the value of the separatrix separating the unsnapped from the snappedthrough configuration in the static load-displacement diagram. We show that the value used to define snap-through is not sensitive for the determination of snap-through region as long as it is near the separatrix. The experimental and numerical snap-through and chaos boundaries show similar features, and confirm the numerical results. Various types of responses are identified: (1) non-chaotic non-snap-through, (2) chaotic non-snap-through, (3) non-chaotic snap-through and (4) chaotic snap-through responses.

For the experimental forcing parameters studied in this paper the persistent snap-through response is always chaotic as opposed to the SDOF system [10] that was very apt to snap-through periodically. This might be because a continuous system requires much higher forces to snap-through in a periodic manner. For the curved beam studied in this paper, periodic and quasiperiodic persistent snap-through were obtained only numerically for much larger forcing amplitudes.

An interesting behavior is observed when the arch snaps-through then oscillates around the remote configuration. During experiments, the arch would frequently get "stuck" in a small amplitude oscillation about the snappedthrough configuration and needed to be perturbed externally for it to snapback. This was also found numerically where a larger force was required to push it out of the snap-through configuration than was originally necessary to cause the initial snap-through event.

\section{Acknowledgements}

The work has been funded in part by AFOSR under the grant no. FA955009-1-0201 and Universal Technology Center contract \#12-S2603. These supports are greatly appreciated. Support was also received from the Data Analysis and Visualization Cyberinfrastructure funded by NSF under grant OCI-0959097. The authors also wish to thank David Ehrhardt for his assistance in the experimental data acquisition. 


\section{References}

[1] B. Zuchowski, Predictive Capability for Hypersonic Structural Response and Life Prediction, Phase 1 - Identification of Knowledge Gaps, Air Vehicles Integration And Technology Research (AVIATR) Task Order 0015 AFRL-RB-WP-TR-2010-3069, Lockheed Martin Aeronautics Company, 2010 .

[2] R. Wiebe, Nonlinear Dynamics of Discrete and Continuous Mechanical Systems with Snap-Through Instabilities, Ph.D. thesis, Duke University, 2012.

[3] G. A. Hegemier, The influence of damping on the snapping of a shallow arch under a step pressure load, Technical Report, California University San Diego, 1969.

[4] N. Huang, Dynamic buckling of some elastic shallow structures subject to periodic loading with high frequency, International Journal of Solids and Structures 8 (1972) 315-326.

[5] W. Y. Poon, C. F. Ng, Y. Y. Lee, Dynamic stability of a curved beam under sinusoidal loading, Proceedings of the Institution of Mechanical Engineers, Part G: Journal of Aerospace Engineering 216 (2002) 209217.

[6] D. A. Ehrhardt, J. L. Abanto-Bueno, C. D. Parks, A. Shukla, Non-contact experimental modal analysis of a curved beam using a full-field optical technique, in: Collection of Technical Papers AIAA/ASME/ASCE/AHS/ASC Structures, Structural Dynamics and Materials Conference (2011).

[7] C. Parks, J. Abanto-Bueno, A. Shukla, Effect of geometric imperfections and boundary conditions on the nonlinear response of a curved beam, in: Collection of Technical Papers - AIAA/ASME/ASCE/AHS/ASC Structures, Conference (2011).

[8] K. D. Murphy, L. N. Virgin, S. A. Rizzi, Characterizing the dynamic response of a thermally loaded, acoustically excited plate, Journal of Sound and Vibration 196 (1996) 635-658. 
[9] C. F. Ng, Nonlinear and snap-through responses of curved panels to intense acoustic excitation, Journal of Aircraft 26 (1989) 281-288.

[10] R. Wiebe, L. N. Virgin, I. Stanciulescu, S. M. Spottswood, T. G. Eason, Characterizing dynamic transitions associated with snap-through: A discrete system, Journal of Computational and Nonlinear Dynamics 8 (2013) 011010.

[11] Y. Chandra, I. Stanciulescu, Virgin, T. G. L. N., Eason, S. M. Spottswood, A numerical investigation of snap-through in a shallow arch-like model, Journal of Sound and Vibration 332 (2013) 2532 2548. http://dx.doi.org/10.1016/j.jsv.2012.12.019i.

[12] R. Plaut, E. Johnson, The effects of initial thrust and elastic foundation on the vibration frequencies of a shallow arch, Journal of Sound and Vibration 78 (1981) 565-571.

[13] R. L. Taylor, University of California, Berkeley. Dept. of Civil and Environmental Engineering, FEAP, a Finite Element Analysis Program: Version 8.3 User Manual (2011).

[14] T. C. Chu, W. F. Ranson, M. A. Sutton, Applications of digital-imagecorrelation techniques to experimental mechanics, Experimental Mechanics 25 (1985) 232-244.

[15] Y. Chandra, I. Stanciulescu, T. Eason, M. Spottswood, Numerical pathologies in snap-through simulations, Engineering Structures 34 (2012) 495-504.

[16] H. M. Hilber, T. J. R. Hughes, R. L. Taylor, Improved Numerical Dissipation for Time Integration Algorithms in Structural Dynamics, Earthquake Engineering and Structural Dynamics 5 (1977) 283-292.

[17] J. Chung, G. Hulbert, Time integration algorithm for structural dynamics with improved numerical dissipation: the generalized- $\alpha$ method, Journal of Applied Mechanics, Transactions ASME 60 (1993) 371-375.

[18] A. Wolf, J. B. Swift, H. L. Swinney, J. A. Vastano, Determining lyapunov exponents from a time series, Physica D 16 (1985) 285-317. 
[19] M. T. Rosenstein, J. J. Collins, C. J. D. Luca, A practical method for calculating largest lyapunov exponents from small data sets, Physica D 65 (1993) 117-134.

[20] R. Wiebe, L. N. Virgin, A heuristic method for identifying chaos from frequency content, Chaos: An Interdisciplinary Journal of Nonlinear Science 22 (2012) 013136-013136.

[21] A. Nayfeh, D. Mook, Nonlinear oscillations, Wiley, 1978.

[22] D. M. N. HaQuang, R. Plaut, A non-linear analysis of the interactions between parametric and external excitations, Journal of Sound and Vibration 118 (1987) 425-439. 


\section{List of Figures}

1 Snap-through buckling of shallow structures: (a) initial and post-snap configurations, (b) force-displacement curve. . . . . 2

2 Photograph of curved arch attached to shaker in clampedclamped configuration; point loading mechanism used for static testing also visible. . . . . . . . . . . . . . 5

3 Schematic of curved arch geometry: (a) pre-clamping geometry, (b) post-clamping geometry, and (c) cross-section. . . . . . 5

4 Illustration of prestressing procedure. (a) Schematic showing axial force and bending moment stages of prestressing. (b) Error norm vs. prestressing end force and end moments.

(c) Design configuration (dashed-dotted line), measured preclamping configuration (empty circles), configuration after application of end force (dashed line, FEA) and end bending moments (solid line, FEA), and measured post-clamping configuration (filled circles). . . . . . . . . . . . 7

5 Comparison of experimental (filled circles) and FEA (solid lines) static force-displacement relationship for midspan point loading: displacement measured at (a) left quarter point, (b) midspan, and (c) right quarter point. (d) Comparison of experimental and FEA natural frequency vs. midspan displacement. * Indicates interpolated displacement measurement. . .

6 Comparison of experimental (filled circles for measured, empty circles for interpolated) and FEA (solid lines) arch configurations at the loads labeled (i) through (iv) in Figure 5 (b). The vertical dashed lines indicate the three locations at which $d_{1}$, $d_{2}^{*}$, and $d_{3}$ were measured. . . . . . . . . . . . . . . . . . . . . 10

$7 \quad$ FEA static force-displacement relationship of prestressed structure (solid lines) and non-prestressed structure (dashed lines) for uniformly distributed loading: displacement measured at (a) left quarter point, (b) midspan, and (c) right quarter point. (d) natural frequency vs. midspan displacement. . . . . . . . 11

8 (a) Static equilibrium path and (b) the stability for the design configuration (circular arch) with distributed loading scenario. Dots in (a) represent unstable solutions. . . . . . . . . . 12

9 Measured post-clamping configuration of arch 1 (filled circles) and 0 (empty circles). . . . . . . . . . . . 13 
10 Experimental velocity (at midspan) time series under harmonic distributed forcing with $A_{F}=8.4 \mathrm{~N} / \mathrm{m}$ for arch 2: (a) frequency sweep from $f_{F}=80$ to $200 \mathrm{~Hz}$ over a period of 80 seconds exhibiting (b) chaotic, (c) period 3, and (d) period 1 response (note the three plots are over different time spans). The superimposed sinusoidal functions (gray) show the forcing frequency (their amplitude is not representative). . . . . . . . 14

11 Experimental peak-to-peak amplitude bifurcation diagrams for arch 0: (a) time integration of velocity (see Figure 10 (b)), (b) sweep up (see Figure 10 (a)), and (c) sweep down bifurcation diagrams . . . . . . . . . . . . . . . . . 15

12 Experimental peak-to-peak amplitude bifurcation diagrams near $f_{F}=115 \mathrm{~Hz}$ bifurcation at two increasing levels of resolution. 16

13 Experimental snap-through rate generated from Figure 12 using different definitions of snap-through. Lighter shading indicates lower snap-through definition. . . . . . . . . . . . . 17

14 Experimental displacement (at midspan) time series under constant frequency harmonic forcing with $A_{F}=1.8 \mathrm{~N} / \mathrm{m}$ for arch 1: (a) $f_{F}=75 \mathrm{~Hz}$ (noisy harmonic non-snap-through response), (b) $f_{F}=130 \mathrm{~Hz}$ (chaotic non-snap-through response ), and (c) $f_{F}=180 \mathrm{~Hz}$ (chaotic snap-through response). DFTs for: (d) $f_{F}=75 \mathrm{~Hz}$, (e) $f_{F}=130 \mathrm{~Hz}$, and (f) $f_{F}=180 \mathrm{~Hz}$. The superimposed sinusoidal function in (a) shows the forcing frequency (the amplitude is not representative). . . . . . . 18

15 Numerical displacement (at midspan) time series under constant frequency harmonic forcing with $A_{F}=1.8 \mathrm{~N} / \mathrm{m}$ for arch 1: (a) $f_{F}=71 \mathrm{~Hz}$ (non-chaotic non-snap-through response), (b) $f_{F}=141 \mathrm{~Hz}$ (chaotic non-snap-through response), and (c) $f_{F}=178 \mathrm{~Hz}$ (chaotic snap-through response). DFTs for: (d) $f_{F}=71 \mathrm{~Hz}$, (e) $f_{F}=141 \mathrm{~Hz}$, and (f) $f_{F}=178 \mathrm{~Hz}$. The superimposed sinusoidal function in (a) show the forcing frequency (the amplitude is not representative). . . . . . . . . 20 
16 (a) Experimental time series showing inverted snap-through (getting "stuck" near the snapped-through configuration) response that needs to be removed with external perturbation, (b) inverted snap-through response obtained numerically, and (c) co-existing chaotic response obtained numerically, (d) inverted snap-through response under a frequency sweep that does not snap-back. . . . . . . . . . . . . . . . . . 21

17 Snap-through and chaos boundaries in forcing amplitude and frequency space obtained through: (a) experiment and (b) numerical simulations for the measured clamped configuration with prestress, (c) the measured clamped configuration without prestress, and (d) the design configuration. The responses identified are snap-through (filled circles), chaotic non-snapthrough (empty circles), and non-chaotic non-snap-through (small dots). The intersections of the dashed gray lines in (a) denote the locations of the three responses shown in Figure 14. The dashed boxes in (b), (c), and (d) indicate the ranges of forcing parameters used in (a). . . . . . . . . . . 22

18 Contour plots for (a) snap-through rate and (b) time required to experience the first snap-through for the prestress structure. 25

19 (a) Snap-through boundaries obtained using different threshold values: $3 \mathrm{~mm}$ (gray) and $6 \mathrm{~mm}$ (black). Area of snapthrough region normalized by the total area for different threshold values for: (b) prestressed structure, (c) non-prestressed structure. (d) Snap-through boundaries obtained using different initial condition (black line indicates the boundary when the arch is excited from equilibrium configuration and gray line indicates boundary obtained when other initial conditions are used). Different initial conditions used expand the snapthrough boundary. . . . . . . . . . . . . . . 26

20 Bifurcation diagram for $A_{F}=3.6 \mathrm{~N} / \mathrm{m} \ldots \ldots$. . . . . . . . . 27

21 Time series for periodic responses: (a) no snap-through $\left(f_{F}=\right.$ $61.5 \mathrm{~Hz})$, (b) inverted snap-through $\left(f_{F}=137 \mathrm{~Hz}\right)$. Phase plots and Poincaré points for (c) no snap-through, (d) inverted snap-through. . . . . . . . . . . . . . . . 28 
22 Time series for quasi-periodic responses: (a) no-snap-through $\left(f_{F}=120.5 \mathrm{~Hz}\right),(\mathrm{b})$ inverted snap-through $\left(f_{F}=158.5 \mathrm{~Hz}\right)$. Poincaré points for (c) no-snap-through, (d) inverted snapthrough. . . . . . . . . . . . . . . . . . 29

23 Time series for chaotic responses: (a) no-snap-through $\left(f_{F}=\right.$ $67.5 \mathrm{~Hz})$, (b) persistent snap-through $\left(f_{F}=143 \mathrm{~Hz}\right) . \quad$. . . . 29

24 Time series for persistent non-chaotic snap-through responses: (a) periodic $\left(A_{F}=22 \mathrm{~N} / \mathrm{m} f_{F}=120 \mathrm{~Hz}\right)$, (b) quasi-periodic $\left(A_{F}=34 \mathrm{~N} / \mathrm{m} f_{F}=120 \mathrm{~Hz}\right)$. (c) Phase plot and Poincaré point that corresponds to (a), (d) Poincaré points that correspond to (b). . . . . . . . . . . . . . 30

\section{List of Tables}

1 Geometry $(\mathrm{mm}) \ldots \ldots \ldots 6$

2 Cross Section and Material Properties . . . . . . . . . 6 